\title{
Mechanism of reversible fluorescent staining of protein with epicocconone
}

Daniel R. Coghlan ${ }^{1}$, James A. Mackintosh ${ }^{1}$ and Peter Karuso ${ }^{1,2^{*}}$

1 FLUOROtechnics Pty Ltd, Sydney, NSW 2109, Australia

2 Department of Chemistry \& Biomolecular Sciences, Macquarie University, Sydney, NSW 2109, Australia

\section{Supplementary Material}

Production of epicocconone (1)

Epicocconone is a naturally occurring azaphilone from the fungus Epicoccum nigrum (AGAL accession number NM 98/00507) that was grown, harvested extracted and purified as previously described (Bell \& Karuso, 2003).

\section{Reaction of epicocconone with amines}

Epicocconone reacts with amines, ammonia, proteins and some amino acids (eg Lys) in a similar fashion to yield a red fluorescent adduct characterized by a fluorescence emission around $610 \mathrm{~nm}$ and a double excitation profile with peaks around 390 and $500 \mathrm{~nm}$ (Fig. S1). This profile is very different to the unreacted epicocconone that fluoresces weekly in the green (Bell \& Karuso, 2003).

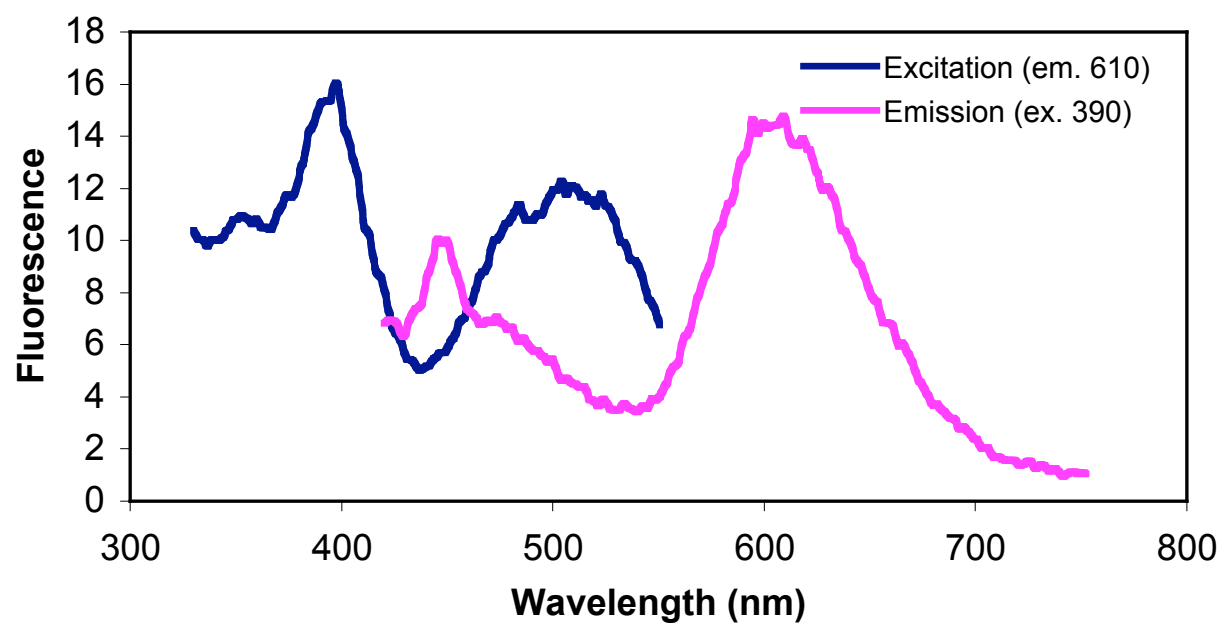

Figure S1. Fluorescence spectrum of epicocconone $(5 \mu \mathrm{g})$ mixed with bovine serum albumin (BSA; 1 $\mathrm{mg} / \mathrm{mL})$, SDS $(0.2 \%)$ and sodium hydroxide $(0.1 \%)$ yields the same fluorescence profile as in 2D gels and upon reaction with butylamine (Fig. 1).

\section{Purification of butylamine adduct}

A solution of epicocconone $(5 \mathrm{mg}, 12 \mu \mathrm{mol})$ in acetonitrile $(1.5 \mathrm{~mL})$ was treated with butylamine $(10$ $\mu \mathrm{L}, 180 \mu \mathrm{mol}$ ) and the resulting pinkish red product purified by reverse phase HPLC (Fig. S2). Using $50 \%$ acetonitrile/water through a Phenomenex Synergi Max-RP column $(10.0 \times 250 \mathrm{~mm}, 10 \mu, 80 \AA)$ at $7 \mathrm{~mL} / \mathrm{min}$, the main component eluted as a pink colored solution after 18 minutes (Fig. S2). The resulting residue was dried under high vacuum overnight to give $\left(S^{*}, Z\right)-6-(($ butylamino)methylene)-5((S*)-2,3-dihydroxypropyl)-3-((1Z,4E,6E,8E)-1-hydroxy-3-oxodeca-1,4,6,8-tetraenyl)-7amethylbenzofuran-2,7 $(6 H, 7 \mathrm{a} H)$-dione $(1 \mathrm{mg}, 17 \%)$ as a red oil.

\footnotetext{
*Corresponding author. Tel.: +612 9850 8290; Fax: +612 9850 8313; e-mail: peter.karuso@mq.edu.au
} 


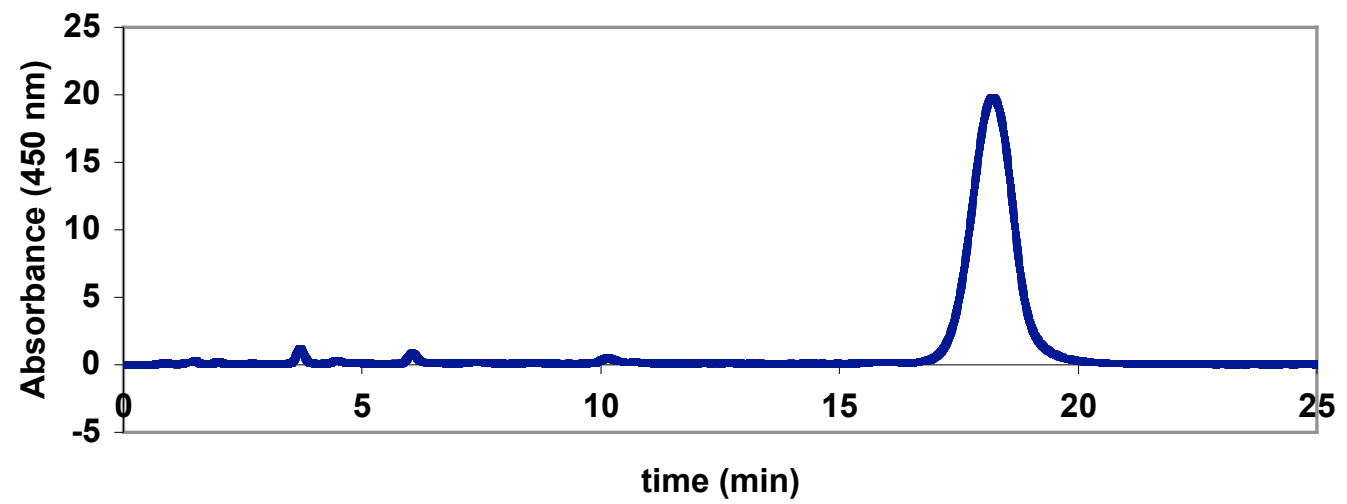

Figure S2. HPLC purification of the crude reaction mixture gave only one peak $\left(\mathrm{A}_{450} \mathrm{~nm}\right)$ by reverse phase gradient elution.

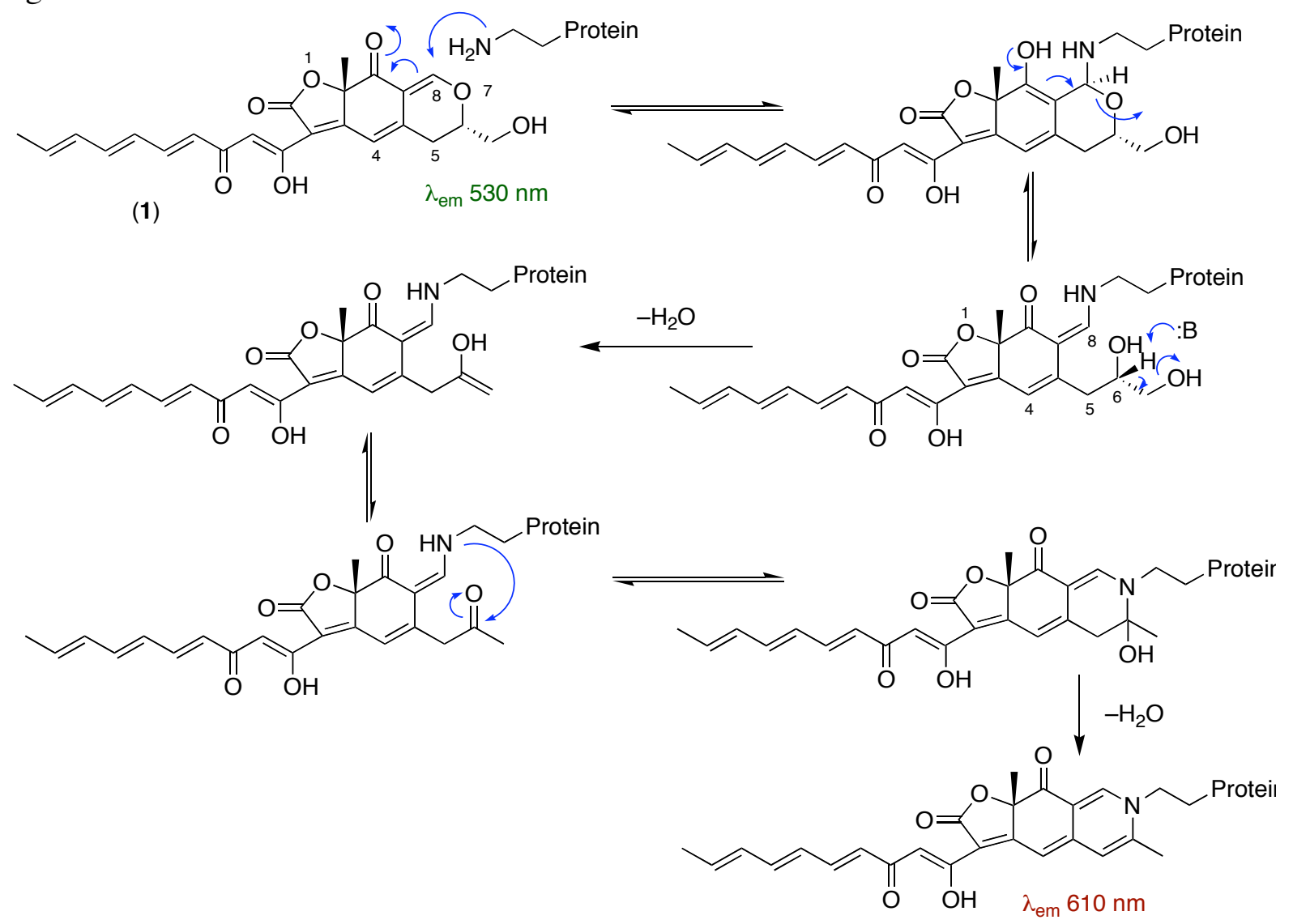

Scheme S1. A possible mechanism for the covalent derivatisation of proteins with epicocconone (1) in the presence of a base that would result in irreversible labeling of proteins. This mechanism is not substantiated by our results.

Initial expectations were that amines reacted with epicocconone via the dihydropyran to form $1 \mathrm{H}$-4pyridylidene as had previously been described for analogous compounds (Scheme S1).

\section{Structure determination}

The structure was determined principally by NMR spectroscopy. 


\section{Nuclear magnetic resonance spectroscopy}

The NMR sample was prepared from the HPLC purified compound $1(\sim 1 \mathrm{mg})$ by dissolving in $\mathrm{CDCl}_{3}$ (0.25 mL; 99.96 atom\%, Aldrich) and filtered into a Shegimi (CMS). NMR tube.

The NMR data were acquired on a Bruker DRX600 (600 MHz) NMR spectrometer at $27^{\circ} \mathrm{C}$ and processed using xwinNMR (version 2.6; Bruker). All 2D NMR experiments were run with quadrature detection with an ${ }^{1} \mathrm{H}$ spectral width of $6009 \mathrm{~Hz}$ and a recycle delay of $2 \mathrm{~s}$. Chemical shifts were referenced to the residual $\mathrm{CHCl}_{3}\left(\delta_{\mathrm{H}} 7.26 \mathrm{ppm} ; \delta_{\mathrm{C}} 77.01 \mathrm{ppm}\right)$. High power ${ }^{1} \mathrm{H} \pi / 2$ pulses were determined to be $9.5 \mu \mathrm{s}$ and low power (for MLEV spin lock) at $25.15 \mu \mathrm{s} .{ }^{13} \mathrm{C}$ high power $\pi / 2$ pulse was $10.5 \mu \mathrm{s}$ and a low power pulse of $65 \mu \mathrm{s}$ was used for GARP decoupling. Gradient pulses were delivered along the z-axis using a 100-step sine program.

Data for $1 \mathrm{D}$ experiments were acquired using $32 \mathrm{~K}$ real points and zero filled to $64 \mathrm{~K}$ and then Gaussian multiplied for resolution enhancement. Carbon - hydrogen correlation (HSQC) was achieved via a sensitivity enhanced double INEPT transfer using echo/antiecho-TPPI gradient (80:20.1) selection (Palmer, 1991; Schleucher, 1994; Kay, 1992). 2K data points were collected in t2 (128 scans per increment) with a $1.3 \mathrm{~s}$ recycle delay with decoupling during acquisition. In $\mathrm{t} 1,512$ increments were used (10-170 ppm) and the INEPT sequence was optimized for a X-H coupling of $145 \mathrm{~Hz}$. A gradient ratio of 80:20.1 was used to select echo/antiecho-TPPI phase sensitivity.

Heteronuclear single quantum coherence (HSQC) was used to correlate and assign all protons to protonated carbons. In addition, each spin system was characterized by running a series of total correlation spectroscopy (TOCSY) experiments with mixing times of $8 \mathrm{msec}, 20 \mathrm{msec}$ and $80 \mathrm{msec}$. The shortest mixing time gave correlations to only the directly coupled protons whereas the longest mixing time identified the entire spin system and gave information about very small long range couplings which were valuable for assigning the positions of the many (apparently) uncoupled protons. Through space connectivities was achieved through a series of one-dimensional selective nuclear Overhauser correlation spectroscopy (NOESY). Selective excitation was achieved by a low-power Gaussian 90 or 270 degree pulse using gradient section to observe only NOE transfers. A mixing time of $200 \mathrm{msec}$ was found optimal.

One-dimensional ROESY spectra were measured using a selective Gaussian pulse on the proton of interest (Kessler, 1986; Stonehouse, 1994; Stott, 1995). A 1000 step Gaussian program (60 ms, 64.6 $\mathrm{dB}$ ) was used to achieve a $\pi / 2$ pulse. A mixing time of $200 \mathrm{~ms}(50 \mathrm{~dB})$ was used for a continuous wave spin lock. Gradient selection was achieved with a $15 \%$ gradient along the $\mathrm{z}$-axis. $10 \mathrm{~K}$ transients were accumulated over $6009 \mathrm{~Hz}$. ROE enhancements were measured as a percentage of the irradiated peak.

Two-dimensional homonuclear Hartman-Hahn transfer spectra (TOCSY) were measured using the MLEV17 (Bax, 1985) pulse sequence flanked with $2 \mathrm{~ms}$ low power trim pulses. Sine bell shifted $\left(90^{\circ}\right)$ apodization was used in the processing of both dimensions.

$2 \mathrm{D}{ }^{1} \mathrm{H}-{ }^{13} \mathrm{C}$ correlation via heteronuclear zero and double quantum coherence optimized for long range couplings (HMBC) with low-pass J-filter $(145 \mathrm{~Hz})$ to suppress one-bond correlations (Bax, 1986) was acquired with no decoupling during acquisition time and using gradient pulses (50:30:40.1) for selection. The delay for evolution of long-range couplings was optimized for couplings of $10 \mathrm{~Hz}$ in separate experiments. A spectral width of 210ppm was used in F1 and the final spectrum magnitude calculated to destroy phase information. 


\section{Mass Spectrometry}

Mass spectra were measured on a Fisons VG Quattro II with a cone voltage of $25 \mathrm{~V}$ and source temperature of $100{ }^{\circ} \mathrm{C}$. High-resolution mass spectra were recorded on a Bruker FT-ICR spectrometer at the University of New South Wales (Gary Willet).

\section{Molecular Modeling}

Molecular dynamics and minimization were performed using the Insight II/Discover3; Molecular Modeling System (Accelrys, 1997). A model of epicocconone-methylamine adduct (Figure S3) was built using the Builder module in InsightII, and energy minimized briefly. Molecular dynamics simulations were performed at $1000 \mathrm{~K}$ for $0.2 \mathrm{~ns}$ and a structure sampled every 10 ps in order to generate 20 random structures. Each structure was cooled to $200 \mathrm{~K}$ over $8 \mathrm{ps}$ using a simulated annealing approach (Proudfoot, 2003). This was followed by minimization of each structure; involving steepest descents, conjugate gradients and quasi-Newton-Raphson minimisation until the final maximum derivative was less than $0.0001 \mathrm{kcal} \mathrm{mol}^{-1} \AA^{-1}$. The cff91 forcefield was used in all Discover3 calculations (Hagler, 1994) and to generate a starting Hessian for the $a b$ initio calculations. All 20 structures for either diasteromer were essentially superimposable.<smiles>C=C1C=CC(=CNC)C(=O)C1</smiles>

Z-isomer<smiles>C=C1C=CC(=CNC)C(=O)C1</smiles>

E-isomer<smiles>C=C1C=CC(/C=N/C)=C(O)C1</smiles>

Z-enol

Figure S3. epicocconone-methylamine adduct starting structures for $a b$ initio calculations.

Starting from the mechanics minimized structures, ab initio geometry and Hessians optimization was performed on the $E$ - and Z-stereoisomers of the epicocconone core (DFT B3LYP/6-31G(d,p)) using Turbomole (Ahrichs, 1989) as implemented by Accelrys (Accelrys, 1997). The optimized geometries and energies (DFT B3LYP/6-31G(d,p)//B3LYP/6-31G(d,p)) are given at the end of this file for the two isomers as well as the enol version of the $Z$-isomer. Given a large enough step size, the geometry optimization of the $Z$-enol always crossed over to the $Z$-isomer. Using an ultrafine grid allowed optimization of the $Z$-enol as reported below. The differences in SCF energies were calculated relative to the $Z$-isomer $(-300791.751 \mathrm{kcal} / \mathrm{mol})$ and ignoring any contribution from zero point energies.

\section{Full Spectral Data}

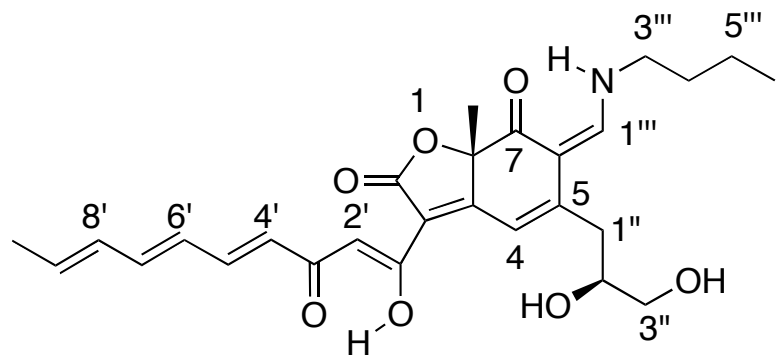

Scheme S2. Numbering pattern used in the numerical NMR data.

MS (ESI +ve) $522\left(18 \%,[\mathrm{M}+\mathrm{K}]^{+}\right), 506\left(32,[\mathrm{M}+\mathrm{Na}]^{+}\right), 484\left(100,[\mathrm{M}+\mathrm{H}]^{+}\right), 466\left(28,\left[\left(\mathrm{M}-\mathrm{H}_{2} \mathrm{O}\right)+\right.\right.$ $\left.\mathrm{H}]^{+}\right), 440\left(28,[(\mathrm{M}-44)+\mathrm{H}]^{+}\right)$.

Daughter ions (MS-MS) of 484: 484 (8\%), 466 (70), 440 (56) and 121 (100).

HR FTIC MS Found 506.214581, $\left[\mathrm{C}_{27} \mathrm{H}_{33} \mathrm{NO}_{7}+\mathrm{Na}^{+}\right]$requires 506.214924. 


\section{UV-VIS}

$\lambda_{\max }$ (acetonitrile) $385,523 \mathrm{~nm}, \log \varepsilon 4.37,4.49$.

${ }^{1} \mathbf{H}$ NMR $\left(600 \mathrm{MHz}, \mathrm{CDCl}_{3}\right)$

$\delta 0.94$, t, J 7.3 Hz, 3H, H6 ',; 1.38, sextet, J 7.5 Hz, 2H, H5 ', ; 1.62, quintet, J 7.2 Hz, H4" ' \& 1.67, s, $5 \mathrm{H}, \mathrm{C} 7 \mathrm{aCH}_{3} ; 1.82$, d $J 6.8 \mathrm{~Hz}, \mathrm{H} 10$ ' \& 1.9, br s, 5H, C2'’'OH and C3'’OH; 2.62, dd, J 5.2, $14.1 \mathrm{~Hz}$, $\mathrm{H}_{2 \mathrm{a}}{ }^{\prime}$, \& 2.66, dd, J 7.2, $14.0 \mathrm{~Hz}, 2 \mathrm{H}, \mathrm{H1}_{2 \mathrm{~b}}{ }^{\prime}$ '; 3.38, q, J $6.6 \mathrm{~Hz}, 2 \mathrm{H}, \mathrm{H} 3{ }^{\prime}$, '; 3.57, dd, J 6.4, $10.9 \mathrm{~Hz}, 1 \mathrm{H}$, $\mathrm{H}_{2 \mathrm{a}}{ }^{\prime}$; 3.76, dd, $J 3.5,10.9 \mathrm{~Hz}, 1 \mathrm{H}, \mathrm{H} 3_{2 \mathrm{~b}}{ }^{\prime}$; $3.96, \mathrm{~m}, 1 \mathrm{H}, \mathrm{H} 2$ ' '; 5.95, dq, J 7, $14.9 \mathrm{~Hz}, 1 \mathrm{H}, \mathrm{H} 9$ '; 6.075, d, $J 15.1 \mathrm{~Hz}, 1 \mathrm{H}, \mathrm{H} 4$ '; 6.18, ddd, $J$ 1.5, 10.8, $14.9 \mathrm{~Hz}, 1 \mathrm{H}, \mathrm{H} 8$ '; 6.24, dd, $J$ 10.8, $14.9 \mathrm{~Hz}, 1 \mathrm{H}, \mathrm{H6}$ '; 6.54, dd, J 10.8, $14.9 \mathrm{~Hz}, 1 \mathrm{H}, \mathrm{H}^{\prime}$ '; 6.76, s, 1H, H2'; 6.92, s, 1H, H4; 7.28, dd, J 11.0, $15.0 \mathrm{~Hz}, 1 \mathrm{H}$, H5'; 7.65, d, J $13.45 \mathrm{~Hz}, 1 \mathrm{H}, \mathrm{H1}{ }^{\prime}$ ' '; 10.41, br dt, J 6.6, $13.5 \mathrm{~Hz}, 1 \mathrm{H}, \mathrm{NH}$; 16.0, br s, 1H, C1'OH.

${ }^{13}$ C NMR (125 MHz, $\left.\mathrm{CDCl}_{3}\right)$

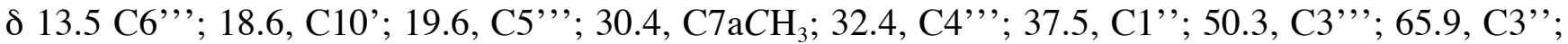
71.6, C2' '; 85.8, C7a; 100.3, C2'; 102.2, C6; 110.3, C4; 111.7, C3, 126.6, C4'; 128.7, C6'; 131.6, C8'; 135.1, C9'; 141.0, C7'; 141.1, C5'; 152.3, C5; 156.1, C1' ' '; 169.5, C2; 173.0, C3a; 180.2, C1'; 182.7, C3'; 194.9 C7. 
From COSY data

\begin{tabular}{|c|c|c|c|c|c|}
\hline 16.0 (br s) & $\mathrm{C} 1{ }^{\prime} \mathrm{OH}$ & & 3.96 & $(\mathrm{mH}) 2^{\prime}$ & \multirow[t]{2}{*}{$\mathrm{H} 1_{\mathrm{a}, \mathrm{b}}}$, \\
\hline 10.4 (br dt) & $\mathrm{NH}$ & $\mathrm{H} 1^{\prime},,, \mathrm{H} 3{ }^{\prime \prime}$, & \multicolumn{2}{|l|}{$\mathrm{H} 3_{\mathrm{a}, \mathrm{b}}}$, & \\
\hline $7.65(d)$ & $\mathrm{H} 1,$, & $\mathrm{NH}$ & $3.76(\mathrm{dd})$ & $\mathrm{H} 3_{2 \mathrm{~b}}$, & $\mathrm{H} 2^{\prime},{ }^{\prime}, \mathrm{H} 3_{2 \mathrm{a}}{ }^{\prime}$, \\
\hline $7.28(\mathrm{dd})$ & $\mathrm{H} 5$ & H6', H4' & $3.57(\mathrm{dd})$ & $\mathrm{H} 3_{2 \mathrm{a}}$, & $\mathrm{H} 2^{\prime}, \mathrm{H}_{2 \mathrm{~b}}{ }^{\prime}$, \\
\hline $6.92(s)$ & H4 & & $3.38(q)$ & H3 ${ }^{\prime},$, & $\mathrm{NH}, \mathrm{H} 4,$, \\
\hline $6.76(\mathrm{~s})$ & $\mathrm{H} 2{ }^{\prime}$ & & $2.66(\mathrm{dd})$ & $\mathrm{H} 1_{2 \mathrm{~b}}$, & $\mathrm{H} 2{ }^{\prime}$ \\
\hline $6.54(\mathrm{dd})$ & $\mathrm{H} 7$ & H6', H8' & $2.62(\mathrm{dd})$ & $\mathrm{H} 1_{2 \mathrm{a}}$, & $\mathrm{H} 2{ }^{\prime}$, \\
\hline $6.24(\mathrm{dd})$ & H6 & $\mathrm{H} 5^{\prime}, \mathrm{H} 7^{\prime}$ & 1.9 (br s) & \multicolumn{2}{|c|}{$\mathrm{C} 2{ }^{\prime \prime} \mathrm{OH} \& \mathrm{C} 3{ }^{\prime \prime} \mathrm{OH}$} \\
\hline $6.18 \mathrm{dd}(\mathrm{l})$ & H8, & H7', H9', & $1.82(\mathrm{~d})$ & H10' & H8', H9' \\
\hline H10' & & & $1.67(\mathrm{~s})$ & $\mathrm{C} 7 \mathrm{aCH} 3$ & \\
\hline $6.07(d)$ & $\mathrm{H} 4{ }^{\prime}$ & $\mathrm{H} 5$ & 1.62 (quin) & $\mathrm{H} 4,$, & $\mathrm{H} 3{ }^{\prime},,, \mathrm{H} 5{ }^{\prime}$, \\
\hline \multirow[t]{2}{*}{$5.95(\mathrm{dq})$} & H9' & H8', H10' & 1.38 (sextet) & $\mathrm{H} 5$, & $\mathrm{H} 4{ }^{\prime}$, , H6", \\
\hline & & & $0.94(t)$ & H6, & $\mathrm{H} 5$, \\
\hline
\end{tabular}

\begin{tabular}{|c|c|c|c|}
\hline${ }^{13} \mathrm{C}$ & & HSQC data & From HMBC data \\
\hline 194.9 & $\mathrm{C} 7$ & & $\mathrm{H} 1{ }^{,},,, \mathrm{H} 4(\mathrm{w}), \mathrm{C}^{2} \mathrm{aCH}_{3}$ \\
\hline 182.7 & C3' & & H5', H2', H4' \\
\hline 180.2 & $\mathrm{C} 1$ & & $\mathrm{H} 2$ \\
\hline 173.0 & $\mathrm{C} 3 \mathrm{a}$ & & $\mathrm{H} 4, \mathrm{C} \mathrm{aCH}_{3}$ \\
\hline 169.5 & $\mathrm{C} 2$ & & \\
\hline 156.1 & $\mathrm{C} 1,$, & & $\mathrm{H} 4, \mathrm{H} 3{ }^{,}$, \\
\hline 152.3 & $\mathrm{C} 5$ & & $\mathrm{H} 1{ }^{\prime},,, \mathrm{H} 4(\mathrm{w}), \mathrm{H} 1{ }^{,}$, \\
\hline 141.1 & $\mathrm{C} 5$ & $7.28 \mathrm{H}^{\prime} / \mathrm{H} 7^{\prime}$ & H9' \\
\hline 141.0 & $\mathrm{C} 7$ & $6.54 \mathrm{H} 5^{\prime} / \mathrm{H} 7^{\prime}$ & H10 \\
\hline 135.1 & C9' & 5.95 H9' & H7', H10' \\
\hline 131.6 & C8, & $6.18 \mathrm{H} 8$ & H7', H6', H10' \\
\hline 128.7 & C6' & $6.24 \mathrm{H} 6$ & $\mathrm{H}^{\prime}{ }^{\prime}(\mathrm{w}), \mathrm{H} 8^{\prime}(\mathrm{w}), \mathrm{H} 4^{\prime}, \mathrm{H} 10^{\prime}(\mathrm{w})$ \\
\hline 126.6 & C4' & $6.07 \mathrm{H} 4{ }^{\prime}$ & $\mathrm{H} 2^{\prime}, \mathrm{H}^{\prime}$ \\
\hline 111.7 & $\mathrm{C} 3$ & & $\mathrm{H} 4, \mathrm{H} 2{ }^{\prime}$ \\
\hline 110.3 & $\mathrm{C} 4$ & $6.92 \mathrm{H} 4$ & $\mathrm{H} 1^{\prime}$ \\
\hline 102.2 & C6 & & $\mathrm{H} 1{ }^{\prime},,(\mathrm{w}), \mathrm{H} 4, \mathrm{H} 1{ }^{\prime}$, \\
\hline 100.3 & $\mathrm{C} 2{ }^{\prime}$ & $6.76 \mathrm{H} 2$ ' & $\mathrm{H} 4$ \\
\hline 85.8 & $\mathrm{C} 7 \mathrm{a}$ & & $\mathrm{H} 1{ }^{\prime},,, \mathrm{H} 4, \mathrm{C} \mathrm{aCH}_{3}$ \\
\hline 71.6 & $\mathrm{C} 2$ ', & 3.96 & $\mathrm{H} 2^{\prime}, \quad \mathrm{H} 3_{2 \mathrm{a}}{ }^{\prime},{ }^{\prime}, \mathrm{H} 1^{\prime}$, \\
\hline 65.9 & C $3{ }^{\prime}$, & $3.76 / 3.52 \mathrm{H} 3^{\prime}$, & $\mathrm{H} 1$, \\
\hline 50.3 & $\mathrm{C} 3,$, & $3.38 \mathrm{H} 3^{,,}$ & $\mathrm{H} 1{ }^{,},, \mathrm{H}_{4},{ }^{\prime}, \mathrm{H} 5$, \\
\hline 37.5 & $\mathrm{C} 1{ }^{\prime}$, & $2.6 \mathrm{H} 1^{\prime}$, & H4, H3”, (w) \\
\hline 32.4 & $\mathrm{C} 4{ }^{\prime \prime}$, & $1.62 \mathrm{H} 4{ }^{\prime,}$, & $\mathrm{H} 3^{,},,{ }^{\prime} 5^{\prime},,, \mathrm{H} 6$, \\
\hline 30.4 & $\mathrm{C} \mathrm{aCCH}$ & $1.67 \mathrm{C}^{2} \mathrm{aCH}_{3}$ & \\
\hline 19.6 & $\mathrm{C} 5$, & $1.38 \mathrm{H} 5$, & H3 ", , H4,", H6", \\
\hline 18.6 & C10' & $1.82 \mathrm{H} 10^{\prime}$ & H8', H9' \\
\hline 13.5 & C6,", & $0.94 \mathrm{H} 6$, & $\mathrm{H} 4{ }^{\prime},,, \mathrm{H} 5{ }^{\prime},$, \\
\hline
\end{tabular}

From TOCSY data 16.0 (br s) $\mathrm{C} 1{ }^{\prime} \mathrm{OH}$

10.4 (br dt)

NHH4,', H5','(w), H6",' (w) 


\begin{tabular}{|c|c|c|c|c|c|}
\hline 7.65 (d) & $\mathrm{H} 1{ }^{\prime \prime}$ & $\begin{array}{l}\text { H3"', H4"', H5", } \\
\text { (w), H6", (w) }\end{array}$ & $\begin{array}{l}3.38(q) \\
2.66(d d)\end{array}$ & $\begin{array}{l}\mathrm{H} 3^{\prime}, \\
\mathrm{H} 1_{2 b},\end{array}$ & $\begin{array}{l}\mathrm{H} 1^{\prime}, ", H 5, ", \mathrm{H}^{\prime}, \\
\mathrm{H} 3_{\mathrm{a}, \mathrm{b}}\end{array}$ \\
\hline $7.28(\mathrm{dd})$ & H5' & $\mathrm{H} 7^{\prime}, \mathrm{H} 8^{\prime}$ & $2.62(\mathrm{dd})$ & $\mathrm{H} 1_{2 \mathrm{a}}{ }^{\prime}$ & $\mathrm{H} 3_{\mathrm{a}, \mathrm{b}}$ \\
\hline $6.92(\mathrm{~s})$ & $\mathrm{H} 4$ & & 1.9 (br s) & $\mathrm{C} 2{ }^{\prime \prime} \mathrm{OH} \& \mathrm{C} 3$ & '”H \\
\hline $6.76(\mathrm{~s})$ & $\mathrm{H} 2$ ' & & $1.82(\mathrm{~d})$ & H10' & $\mathrm{H} 7$ \\
\hline $6.54(\mathrm{dd})$ & $\mathrm{H} 7$ & H5', H9', H10' & $1.67(\mathrm{~s})$ & $\mathrm{C} 7 \mathrm{aCH} 3$ & \\
\hline $6.24(\mathrm{dd})$ & H6' & H9' & 1.62 (quin & )H4,", & $\mathrm{NH}, \mathrm{H} 1$ ",', H6", \\
\hline 6.18 (ddd) & $\mathrm{H} 8{ }^{\prime}$ & & $1.38(\mathrm{sexte}$ & & H5", $\quad$ H3",', \\
\hline $6.07(\mathrm{~d})$ & $\mathrm{H} 4$ & & & & $\mathrm{H} 1$ ", (w), NH (w) \\
\hline $5.95(\mathrm{dq})$ & H9' & $\mathrm{H} 6{ }^{\prime}, \mathrm{H} 7{ }^{\prime}$ & $0.94(t)$ & H6", & $\mathrm{H} 4$ "', H3",', H1"," \\
\hline $3.96(\mathrm{~m})$ & $\mathrm{H} 2$ ', & & & & (w), NH (w) \\
\hline $3.76(\mathrm{dd})$ & $\mathrm{H} 3_{2 b}$, & $\mathrm{H} 1_{\mathrm{a}, \mathrm{b}}$ & & & \\
\hline $3.57(\mathrm{dd})$ & $\mathrm{H} 3_{2 \mathrm{a}}$, & $\mathrm{H} 1_{\mathrm{a}, \mathrm{b}}$ & & & \\
\hline
\end{tabular}

From ROSEY data

Connectivity between $\mathrm{H} 1$ ', , $\mathrm{H} 2$ ' ' and $\mathrm{H} 1$ ',

\section{Hydrolysis rates by Mass spectrometry}

Epicocconone ( $25 \mathrm{nmol})$ was added to each of the amino acids $(25 \mathrm{nmol})$ : arginine, asparagine, aspartic acid, cysteine, glycine, histidine, lysine, methionine, proline and serine in a ratio of approximately 1:1 in carbonate buffer $(10 \mathrm{mM} ; 100 \mu \mathrm{L} ; \mathrm{pH} \sim 10)$ for 30 minutes. All the samples had a greenish yellow fluorescence, due to epicocconone, apart from the basic amino acids, which fluoresced orange to red; lysine had the strongest red fluorescence. Each sample was diluted 1:10 into 0.1\% formic acid, 50\% acetonitrile and injected into an ESI-MS. Analysis of the reaction mixtures showed that only the basic amino acids had a signal corresponding to the epicocconone addition product (Figure S4) with lysine being the strongest.

Synthetic formaecin 1 (unglycosylated form; $118 \mathrm{nmol}$ ) was combined with epicocconone (200 nmol) in 50:50 DMSO/acetonitrile was dissolved in $4 \mathrm{mM}$ sodium carbonate. Samples were incubated at 26 ${ }^{\circ} \mathrm{C}(1.5 \mathrm{~h})$ in the dark and $10-\mu \mathrm{L}$ aliquots were removed and added to $90 \mu \mathrm{L}$ of various concentrations of acid (Table S1) and immediately mixed $1 \mu \mathrm{l}$ with $1 \mu \mathrm{L}$ of freshly recrystallized $\alpha$-Cyano-4hydroxycinnamic acid $(8 \mathrm{mg} / \mathrm{mL}$ in $70 \%$ Acetonitrile $0.1 \%$ TFA) and quickly dried (nitrogen) onto a MALDI target in duplicate. Mass spectra were recorded immediately (TOFSpec 2E, Micromass) and samples were then stored in the dark at $21{ }^{\circ} \mathrm{C}$ and sampling was repeated, as described, after $2 \mathrm{~h} 15$ min, $20 \mathrm{~h} 15 \mathrm{~min}, 44 \mathrm{~h}$, and $116 \mathrm{~h}$. The integral for the formaecin 1 molecular ion cluster centered at $1794 \mathrm{amu}$ was normalized to $100 \%$ and the integral of the epicocconone adduct (2204 amu) cluster compared in each run. Averages of replicate runs were calculated and graphed (Figure S5) and fitted to a standard exponential, assuming a pseudo-first order decay. The first order rate constants (Table S1) were graphed against $\mathrm{pH}$ (see Figure 4 in the paper). 
Lysine Epi 2 MS

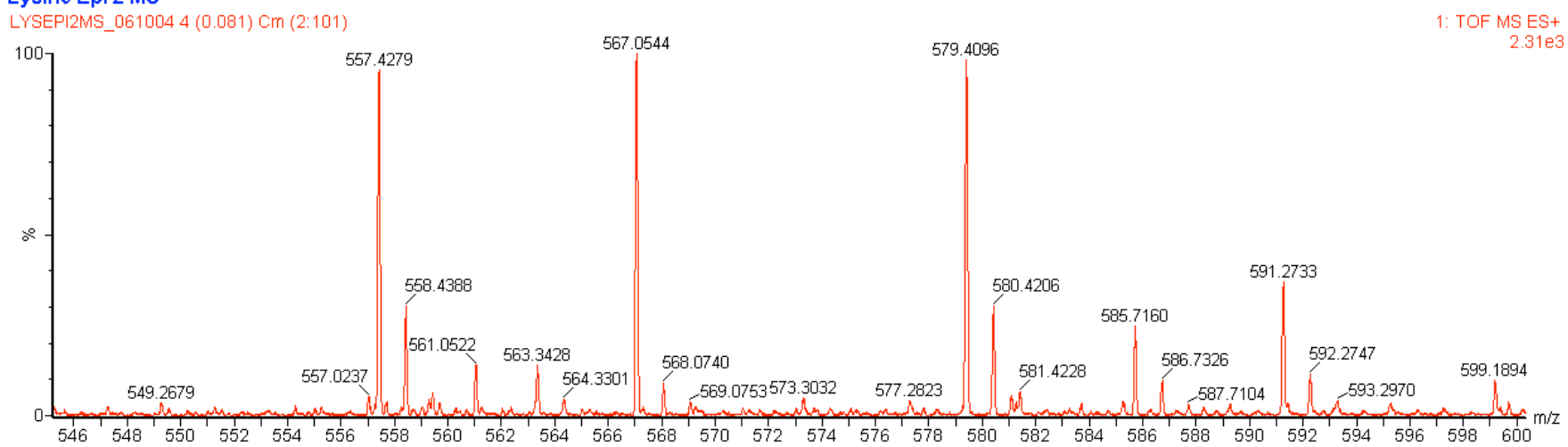

ARG Epi 2 MS repeat

ARGEPI2MS_071004 85 310) Cm (2:105)

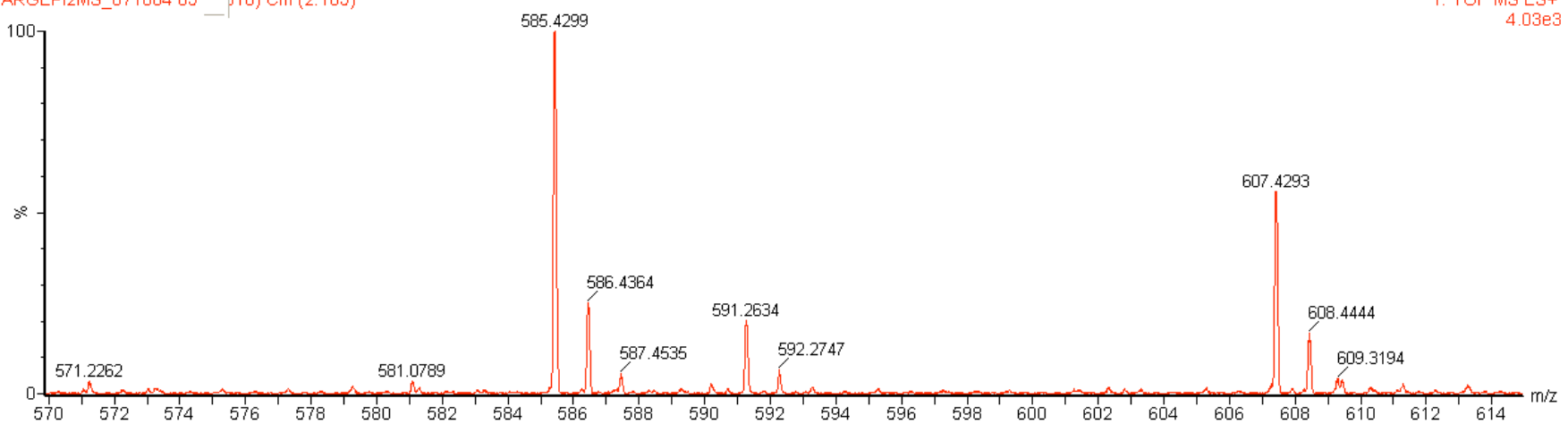

Histidine Epi 2 MS

HISEPI2MS_061004 51 (0.965) Cm (2:116)

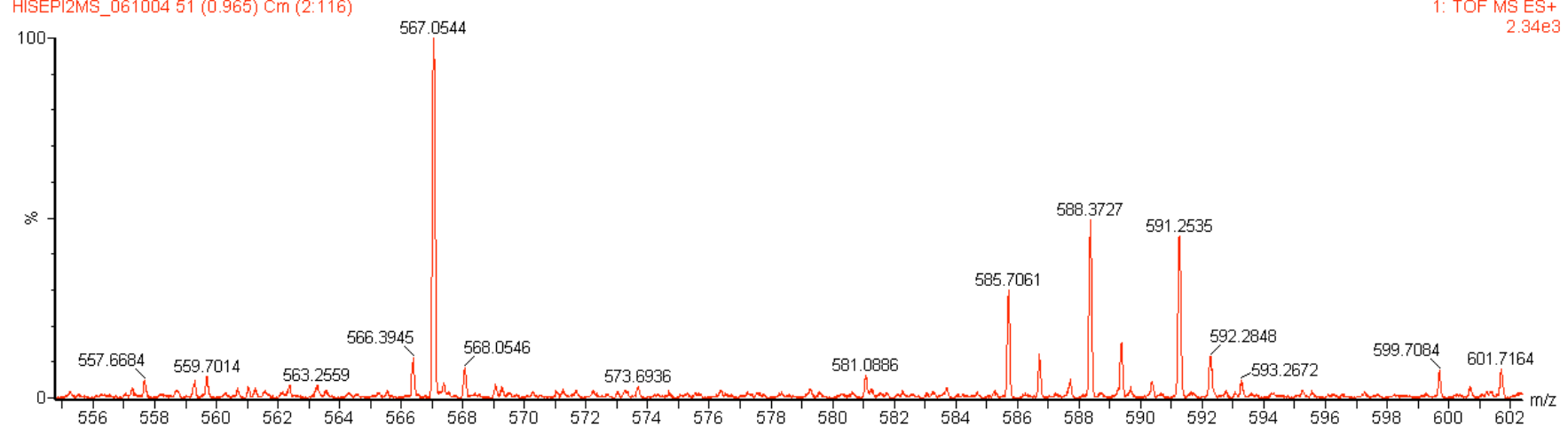



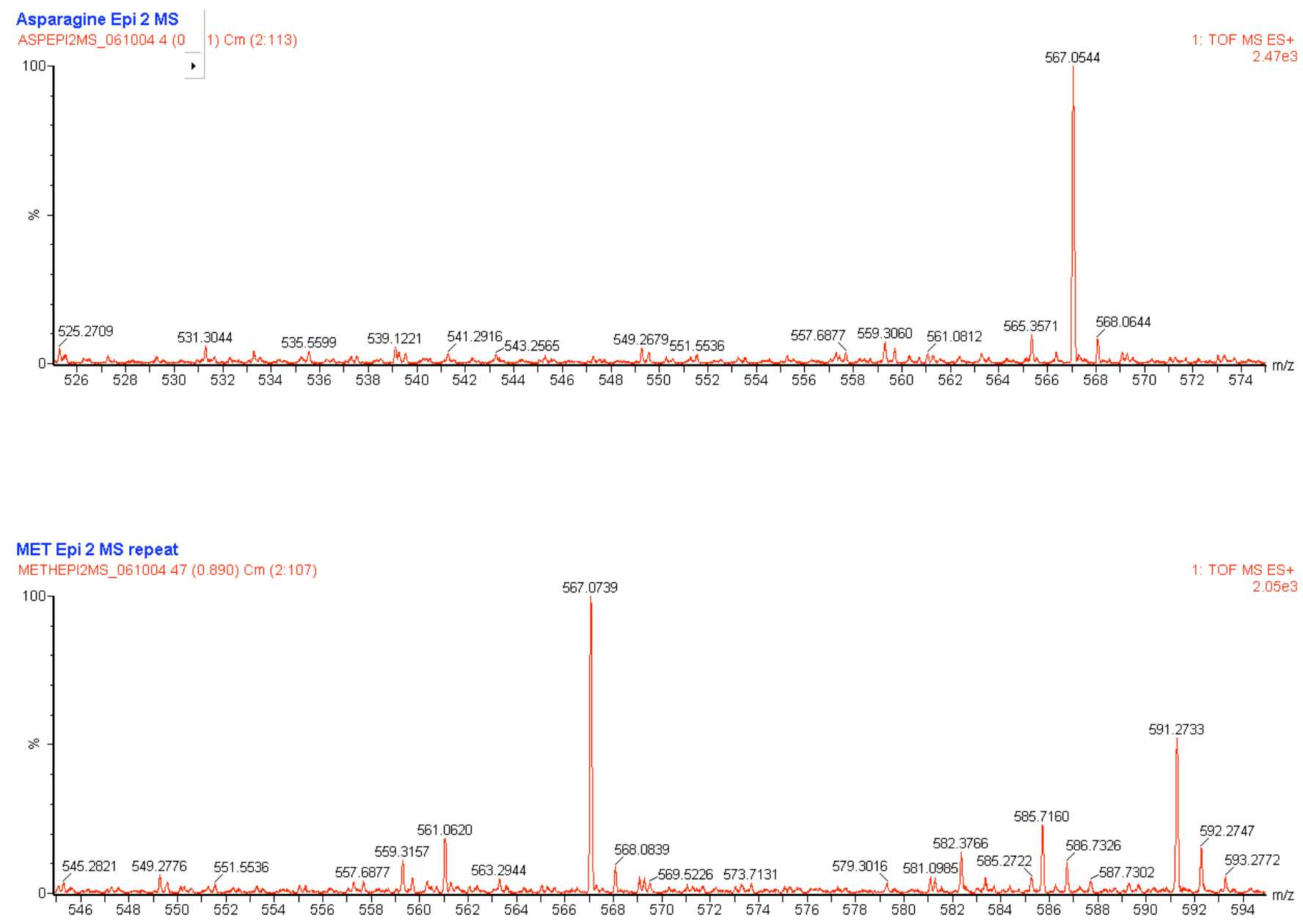

Figure S4. MALDI MS spectra of lysine, arginine, histidine, asparagine and methionine mixed 1:1 with epicocconone. Note the signal at 567.1 is due to matrix. [Lys+epi+H $\left.\mathrm{H}^{+}\right]=557$, $\left[\mathrm{Lys}+\mathrm{epi}+\mathrm{Na}^{+}\right]=$ 579; $\left[\mathrm{Arg}+\mathrm{epi}+\mathrm{H}^{+}\right]=585,\left[\mathrm{Arg}+\mathrm{epi}+\mathrm{Na}^{+}\right]=607 ;\left[\mathrm{His}+\mathrm{epi}+\mathrm{H}^{+}\right]=566,\left[\mathrm{His}+\mathrm{epi}+\mathrm{Na}^{+}\right]=588$; $\left[\right.$ Asn+epi+H $\left.{ }^{+}\right]=543,\left[\right.$ Asn+epi+Na $\left.{ }^{+}\right]=565 ;\left[\mathrm{Met}+\right.$ epi+H $\left.\mathrm{H}^{+}\right]=560,\left[\mathrm{Met}+\mathrm{epi}+\mathrm{Na}^{+}\right]=582$. 
Table S1. Hydrolysis rates of formaecin 1 in various solvents as measured by the ratio of formaecin 1 to formaecin-epicocconone adduct measured over 144 hours by MALDI-MS.

\begin{tabular}{|l|c|l|}
\hline Solvent & Measured $\mathrm{pH}$ of solvent & Rate of hydrolysis* \\
\hline Pure water & 6.00 (estimated) & 2.817 \\
$0.01 \%$ Acetic acid & 3.73 & 0.101 \\
$0.05 \%$ Acetic acid & 3.42 & 0.042 \\
$0.1 \%$ Acetic acid & 3.21 & 0.040 \\
$0.01 \%$ TFA & 2.90 & 0.034 \\
$0.5 \%$ Acetic acid & 2.89 & 0.014 \\
$1.0 \%$ Acetic acid & 2.72 & 0.017 \\
$5.0 \%$ Acetic acid & 2.40 & 0.015 \\
$0.05 \%$ TFA & 2.29 & 0.017 \\
$0.1 \%$ TFA & 2.04 & 0.025 \\
$0.5 \%$ TFA & 1.59 & 0.043 \\
$1.0 \%$ TFA & 1.00 (calculated) & 0.101 \\
\hline
\end{tabular}

* rates of hydrolysis were calculated using Graphpad Prism, fitting the decay of $\mathrm{f}(t)=\mathrm{c}+e^{-\mathrm{k} t}$. All $\mathrm{R}^{2}$ values were better than 0.9 .

Clearly the rate of hydrolysis is $\mathrm{pH}$ dependant and acid independent, suggesting a normal base catalyzed and acid catalyzed (at less than $\mathrm{pH} 2)$ mechanism apply. Thus the overall rate $\left(k_{\mathrm{O}}{ }^{\prime}\right)$ can be made up of three components representing the rate of hydrolysis by water $\left(k_{\mathrm{W}}{ }^{\prime}\right)$, hydronium $\left(k_{\mathrm{A}}{ }^{\prime}\right)$ and hydroxide $\left(k_{\mathrm{B}}{ }^{\prime}\right)$ ions:

$$
\begin{aligned}
& k_{O}{ }^{\prime}=k_{W}{ }^{\prime}+k_{A}{ }^{\prime}\left[H_{3} O^{+}\right]+k_{B}{ }^{\prime}\left[O H^{-}\right] \\
& k_{O}{ }^{\prime}=k_{W}{ }^{\prime}+k_{A}{ }^{\prime}\left[H_{3} O^{+}\right]+k_{B}{ }^{\prime}\left(\frac{K_{W}}{\left[H_{3} O^{+}\right]}\right) \\
& k_{O}{ }^{\prime}=k_{W}{ }^{\prime}+k_{A}{ }^{\prime} 10^{-p H}+k_{B}{ }^{\prime} 10^{(p H-14)}
\end{aligned}
$$

Initially setting the value of $k_{\mathrm{w}}$ ' to zero, the equation was solved using least squares regression (Prism, 2004). An $\mathrm{R}^{2}$ value of 0.93 was obtained which was improved slightly (0.96) by adding $k_{\mathrm{w}}$ as a variable.

$\begin{array}{ll}\text { pH } & \text { rate } \\ 3.7 & 0.1016720 \\ 3.4 & 0.0419037 \\ 3.2 & 0.0408379 \\ 2.9 & 0.0338656 \\ 2.7 & 0.0168552 \\ 2.4 & 0.0155474 \\ 2.3 & 0.0173912 \\ 2.0 & 0.0251797 \\ 1.6 & 0.0429637 \\ 1.0 & 0.1007929\end{array}$
Best-fit values
$k_{\mathrm{W}}{ }^{\prime} \quad 0.01154$
$k_{\mathrm{H}}, \quad 0.91105$
$k_{\mathrm{B}}^{\prime} \quad 1.693 \mathrm{e}+9$
Std. Error
$k_{\mathrm{W}}, \quad 0.003375$
$k_{\mathrm{H}}, \quad 0.08311$
$k_{\mathrm{B}}, \quad 1.610 \mathrm{e}+8$
95\% Confidence Intervals
$\begin{array}{ll}k_{\mathrm{W}}, & 0.003561 \text { to } 0.01953 \\ k_{\mathrm{H}}, & 0.9000 \text { to } 1.107 \\ k_{\mathrm{B}}, & 1.312 \mathrm{e}+009 \text { to } 2.074 \mathrm{e}+009\end{array}$
Goodness of Fit

$\mathrm{R}$ squared

0.9607 

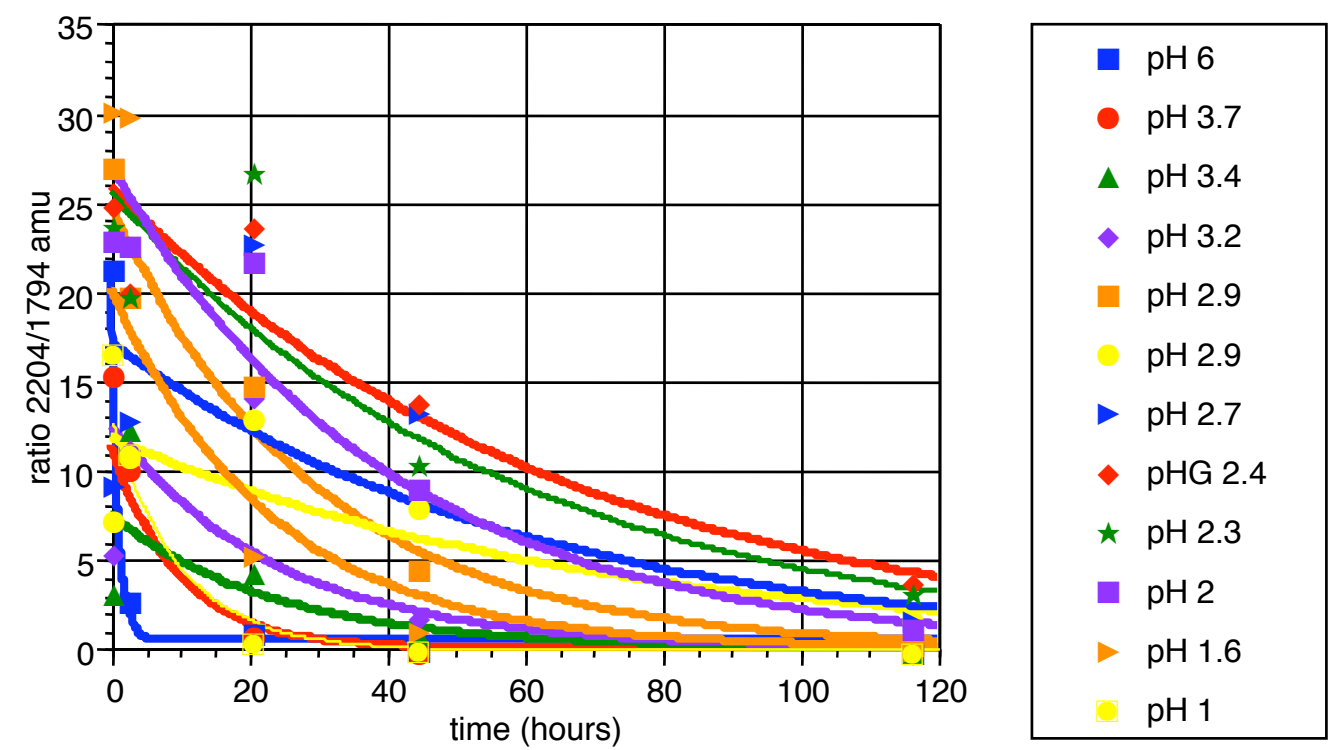

Figure S5. Least squares fit of the rates of hydrolysis of formaecin 1 aglycone-epicocconone adduct over 144 hours as measured by the relative integrals of molecular ion clusters of formaecin and the epicocconone adduct by MALDI-MS.

\section{References}

Accelrys, in 'InsightII/Discover3 Molecular Modeling System', San Diego, USA, 1997.

Ahlrichs, R., Bär, M., Häser, M., Horn, H. \& Kölmel, C. (1989) Chem. Phys. Lett., 162, 165.

Bax, A., \& Davis, D. G. (1985) J. Magn . Reson. 63, 207-13.

Bax, A., \& M.F. Summers, (1986) J. Am. Chem. Soc. 108, 2093-2094.

Bell, P. J. L.; Karuso, P. J. Am. Chem. Soc. 2003, 125, 9304-5.

Prism version 4, Graphpad Software, 2004

Hagler, A. T., \& Ewig, C. S., (1994) Comp. Phys. Chem., 84, 131-155.

Kay, L. E., Keifer, P., and Saarinen, T. (1992) J. Am. Chem. Soc. 114, 10663-5.

Kessler, H., H. Oschkinat, C. Griesinger \& W. Bermel, (1986) J. Magn. Reson. 70, 106.

Palmer, A. G., III, Cavanagh, J., and Wright, P. (1991) J. Magn. Reson. 93, 151-70

Proudfoot, E. M., Mackay, J. P. \& Karuso, P. (2003) Dalton Trans., (2), 165-170.

Schleucher, J., Schwendinger, M., Sattler, M., and Schmidt, P. (1994) J. Biomol. NMR 4, 301-6.

Stadler, M., Anke, H., Dekermendjian, K., Reiss, R., Sterner, O., \& Witt, R. (1995) Nat. Prod. Lett. 7, 7-14.

Stonehouse, J., P. Adell, J. Keeler \& A.J. Shaka, (1994) J. Am. Chem. Soc. 116, 6037.

Stott, K., J. Stonehouse, J. Keeler, T.L. Hwang \& A.J. Shaka, (1995) J. Am. Chem. Soc. 117, 4199-4200. 


\section{Epi-BuNH2}

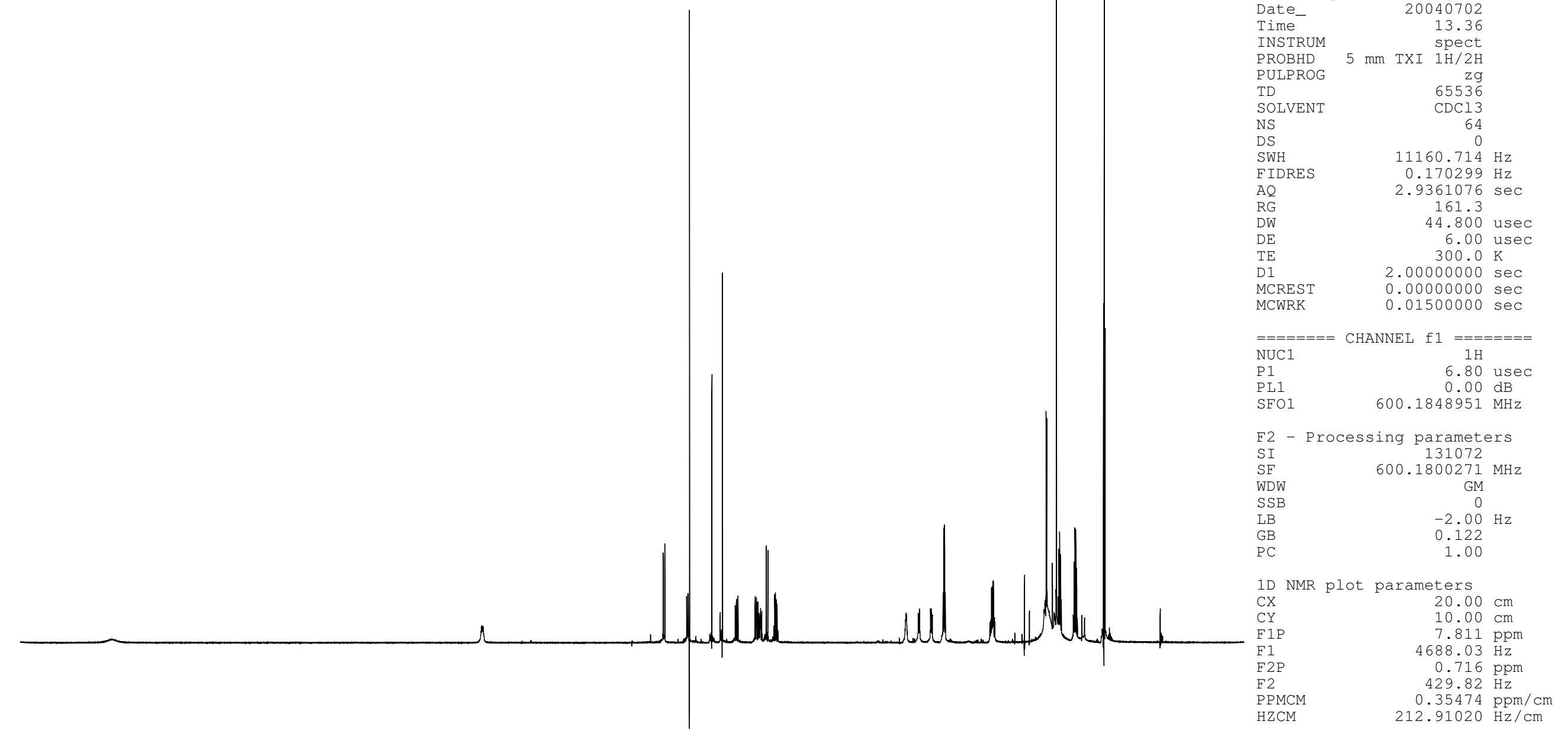


HPLC purified butylamine derivatisd epicocconone (8) after HPLC (lower trace) and after 12 months in CDCl3. Note the appearance of singlets for H4 (7.13 ppm) and $\mathrm{H} 8(8.75 \mathrm{ppm})$ indicative of the slow conversion back to epicocconone.

1h NMR epi-butylamine HPLC purified
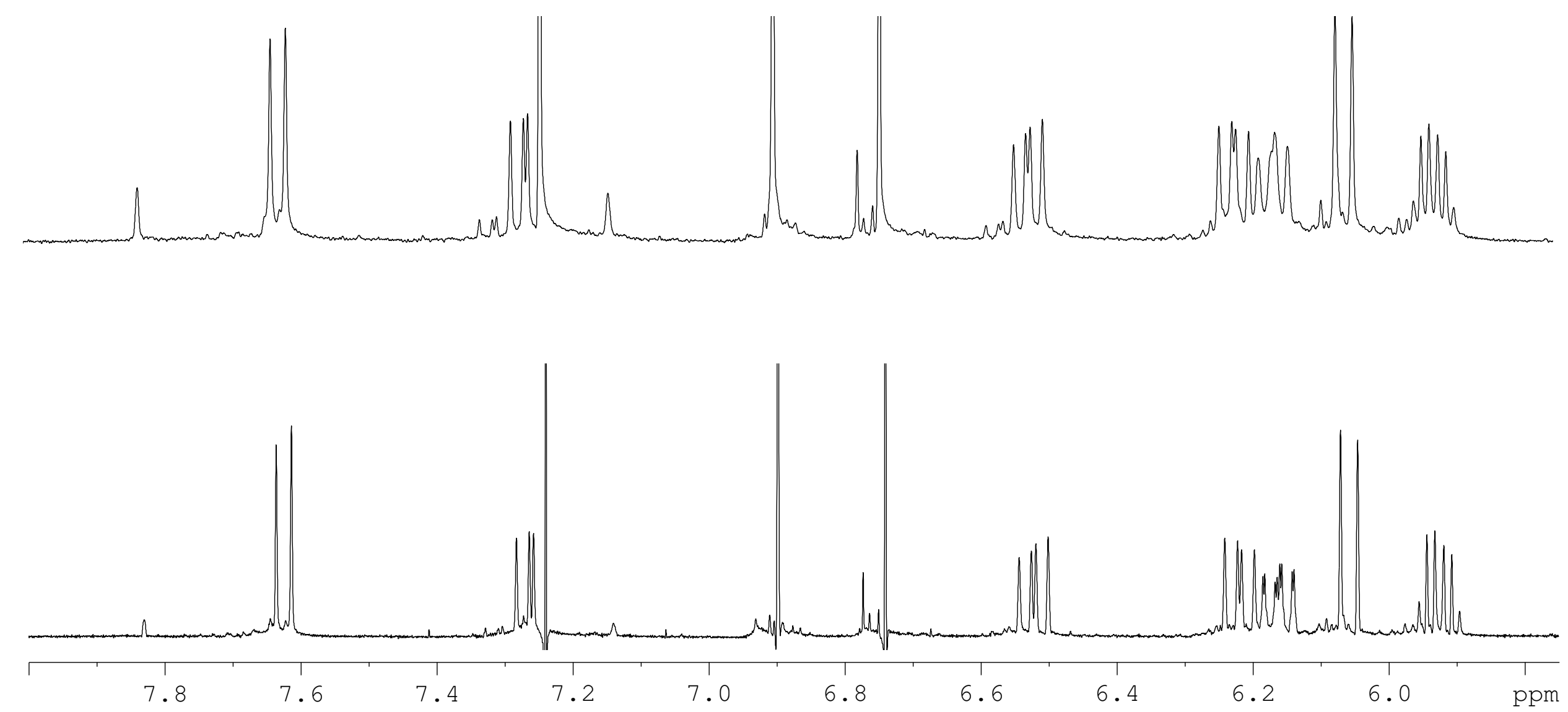


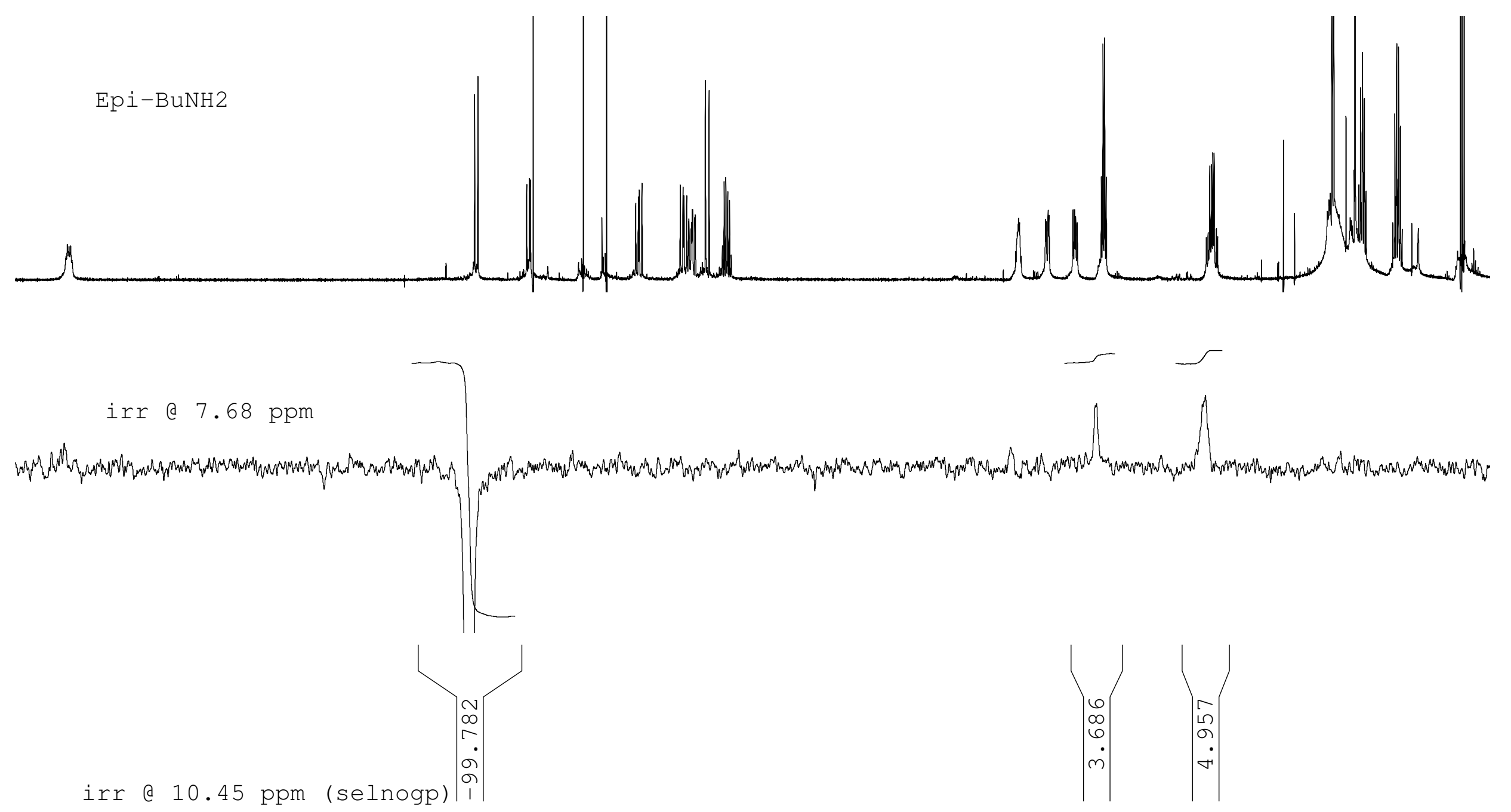

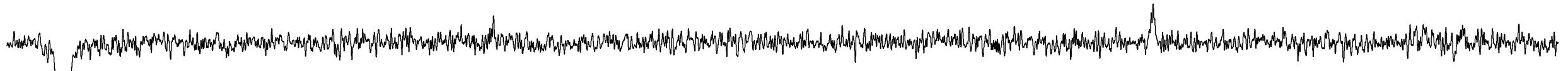

Selective irradiation of $\mathrm{H} 8$ (middle) and $\mathrm{NH}$ (lower) protons and measurement of a selective nOe. 

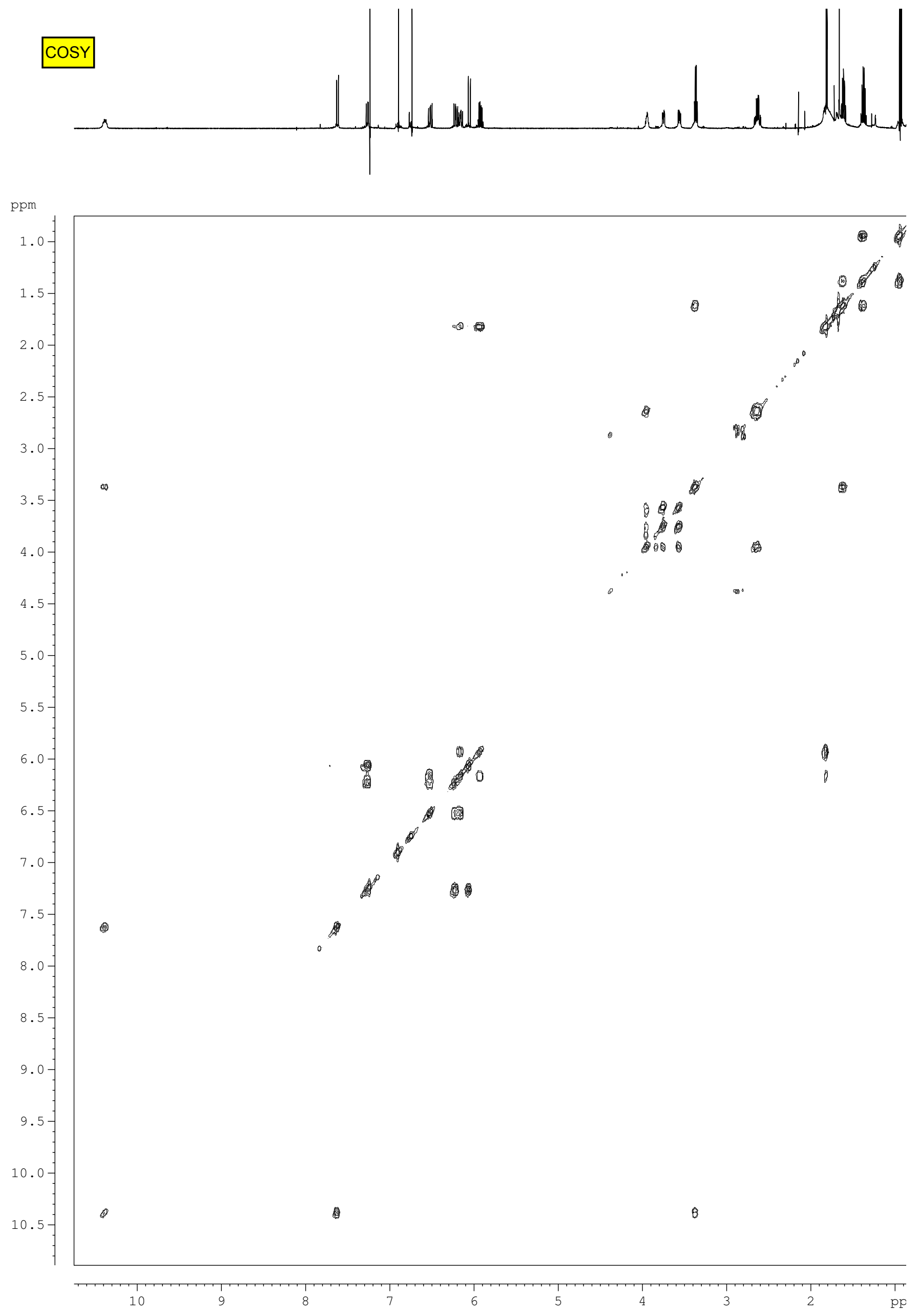


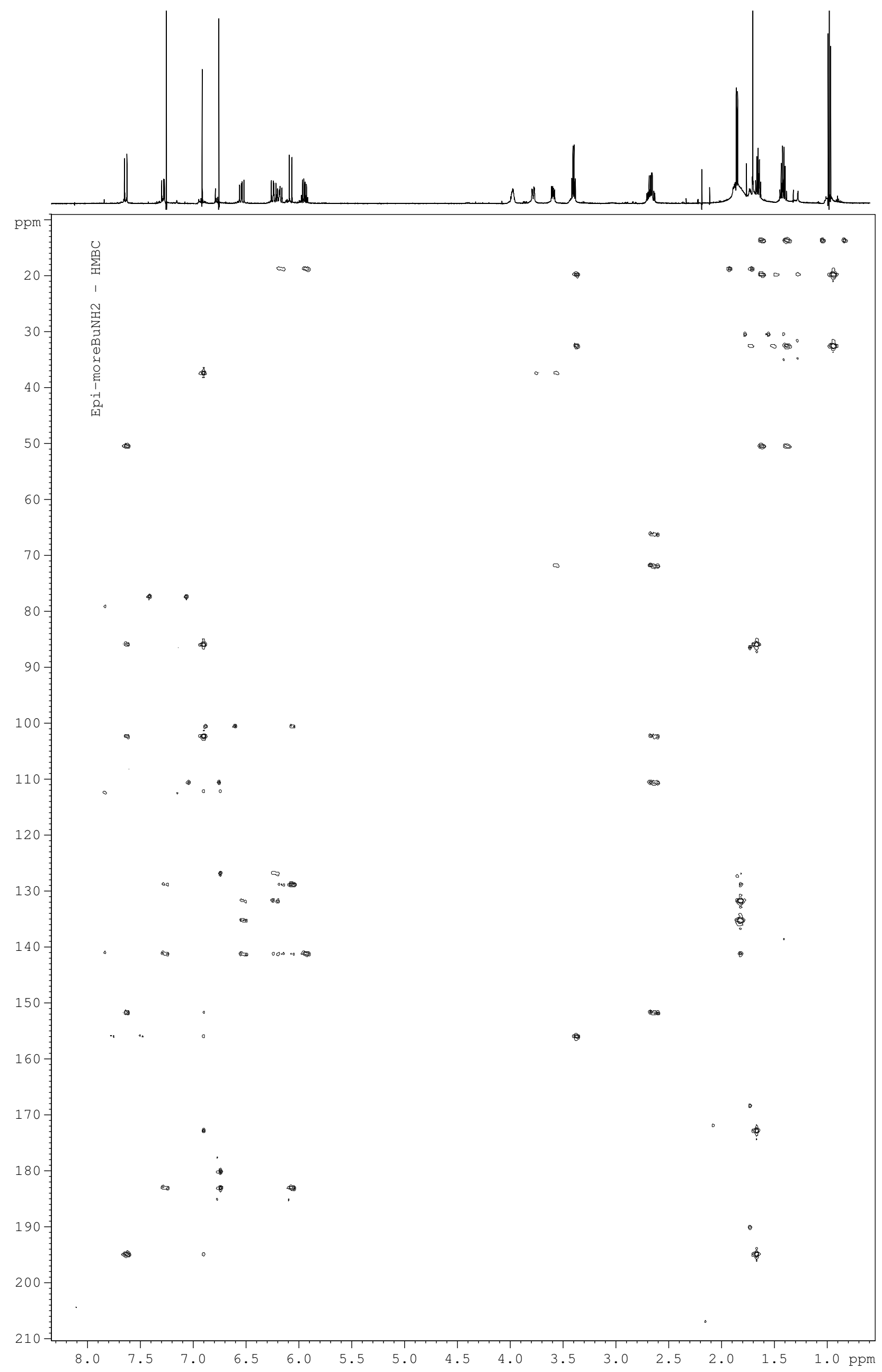




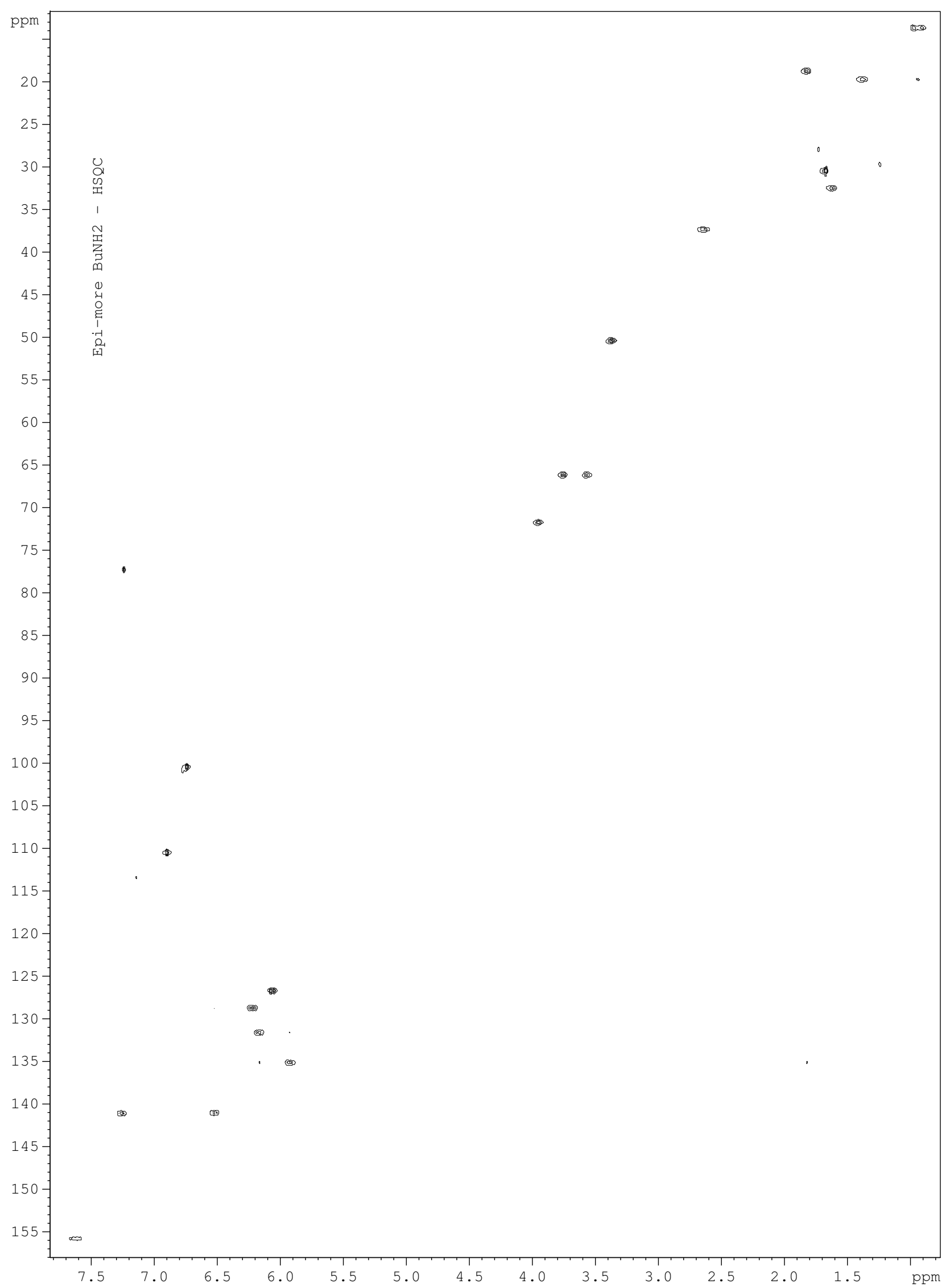




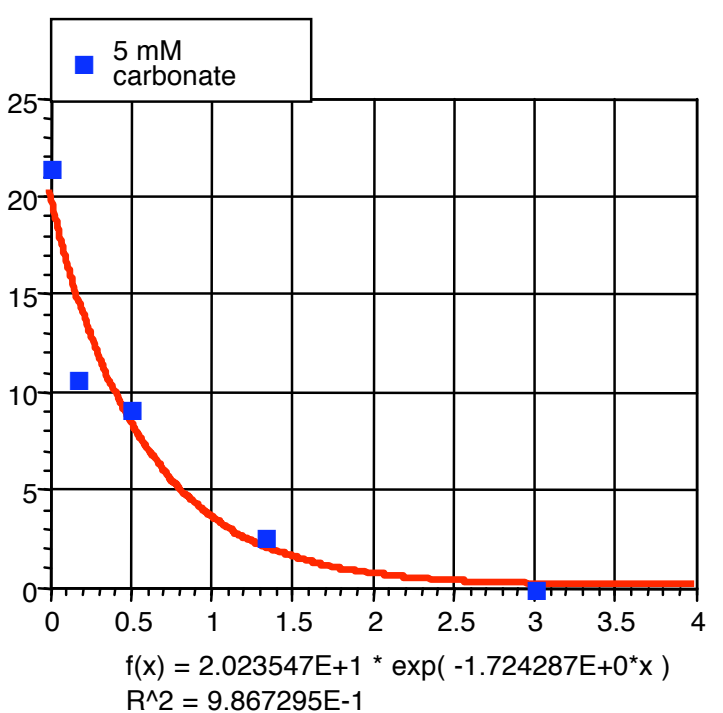

$0.005 \%$ TFA

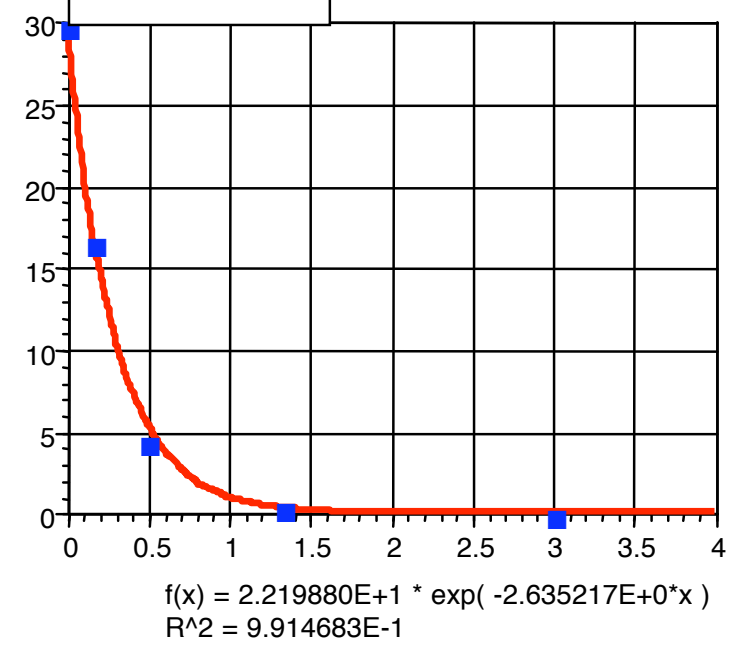

$0.05 \%$ TFA

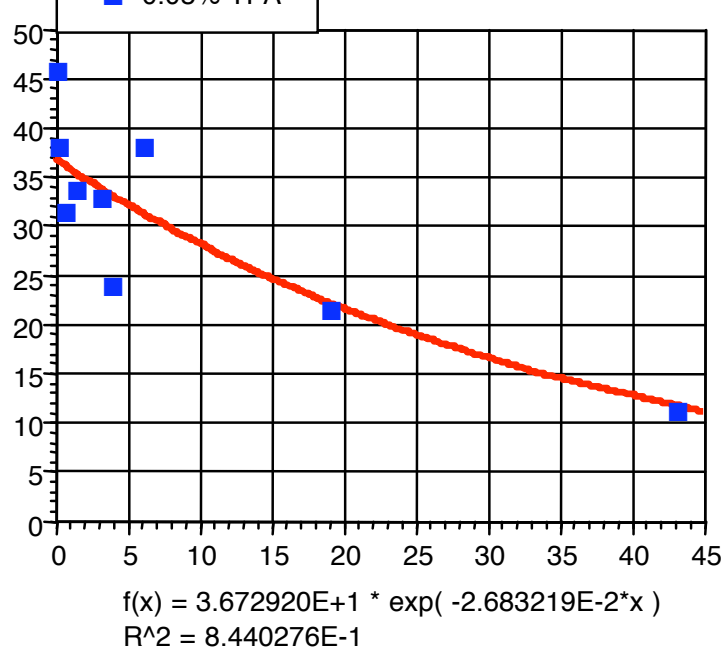

$\mathrm{R}^{\wedge} 2=8.440276 \mathrm{E}-1$

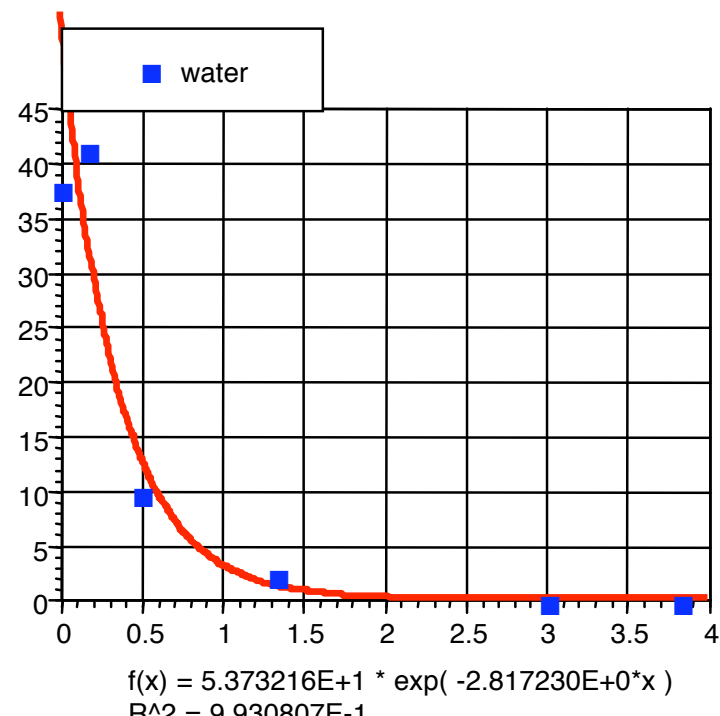

$f(x)=5.373216 \mathrm{E}+1^{*} \exp \left(-2.817230 \mathrm{E}+0^{*} \mathrm{x}\right)$

$\mathrm{R}^{\wedge} 2=9.930807 \mathrm{E}-1$

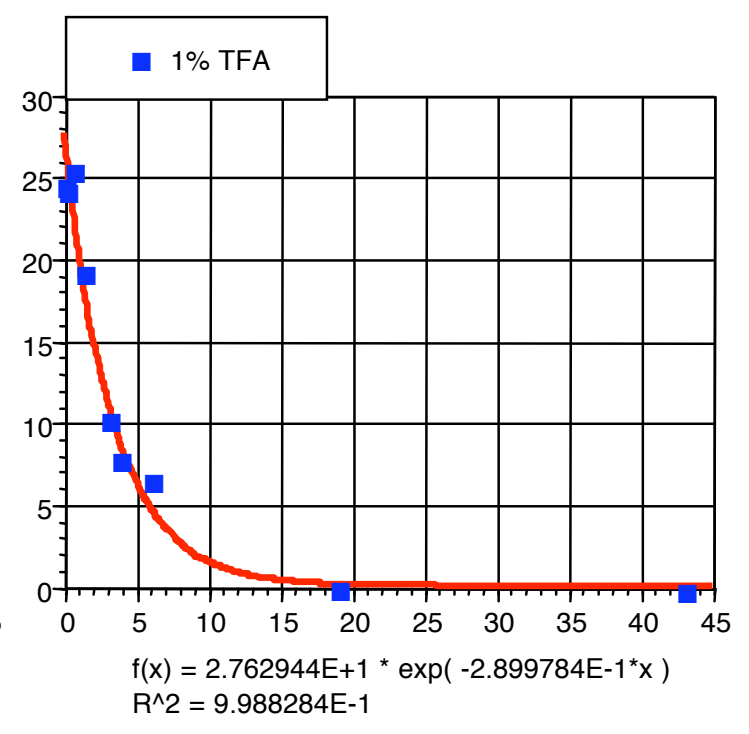

Calculation of rate of hydrolysis based on least-squares fit to an exponential decay:

$f(t)=c+e^{-k t}$ 
Product: turbomole

Job Name: z-enol_finalo

Calculation Type: Optimize

Created: Sat Dec 18 08:37:10 2004

$\begin{array}{lll}\text { Method: dft } & \text { Basis: } 6-31 g^{* *} & \text { Symmetry: cs } \\ \text { Spin: RESTRICTED } & \text { Charge: } 0 & \text { Multiplicity: } 1 \\ \text { Functionals: blyp } & \text { Grid: xfine } & \end{array}$

\#\#\#\#\#\#\#\#\#\#\#\#\#\#\#\#\#\#\#\#\#\#\#\#\#\#\#\#\#\#\#\#\#\#\#\#\#\#\#\#\#\#\#\#\#\#\#\#\#\#\#\#\#\#\#\#\#\#\#\#\#\#\#\#\#

Optimized Molecular Geometry

\begin{tabular}{|c|c|c|c|c|c|c|}
\hline \multirow[b]{2}{*}{ Atom } & \multicolumn{2}{|c|}{ Cartesian Coordinates } & (Angstrom) & \multicolumn{3}{|c|}{ Cartesian Gradients (au) } \\
\hline & $x$ & $\mathrm{y}$ & z & $d E / d x$ & $d E / d y$ & $d E / d z$ \\
\hline---- & & & & & & \\
\hline $1 \mathrm{C}$ & -1.787640 & -1.108515 & 0.000000 & 0.000151 & -0.000105 & 0.000000 \\
\hline $2 C$ & -0.254180 & -0.975979 & 0.000000 & -0.000123 & -0.000010 & 0.000000 \\
\hline $3 C$ & 0.336149 & 0.361573 & 0.000000 & 0.000130 & -0.000101 & 0.000000 \\
\hline $4 C$ & -0.541563 & 1.525935 & 0.000000 & -0.000016 & 0.000013 & 0.000000 \\
\hline $5 \mathrm{C}$ & -1.904915 & 1.447868 & 0.000000 & -0.000140 & 0.000101 & 0.000000 \\
\hline $6 \mathrm{C}$ & -2.623110 & 0.178976 & 0.000000 & -0.000016 & 0.000155 & 0.000000 \\
\hline 70 & 0.430670 & -2.032964 & 0.000000 & -0.000074 & -0.000069 & 0.000000 \\
\hline $8 \mathrm{H}$ & 2.160607 & -1.435285 & 0.000000 & 0.000035 & 0.000003 & 0.000000 \\
\hline $9 \mathrm{C}$ & 1.727387 & 0.514913 & 0.000000 & 0.000114 & -0.000013 & 0.000000 \\
\hline $10 \mathrm{H}$ & 2.157248 & 1.524380 & 0.000000 & -0.000036 & -0.000051 & 0.000000 \\
\hline $11 \mathrm{~N}$ & 2.612437 & -0.504315 & 0.000000 & -0.000244 & 0.000047 & 0.000000 \\
\hline $12 \mathrm{C}$ & 4.063785 & -0.333114 & 0.000000 & -0.000035 & -0.000034 & 0.000000 \\
\hline $13 \mathrm{C}$ & -3.980610 & 0.121762 & 0.000000 & 0.000049 & 0.000009 & 0.000000 \\
\hline $14 \mathrm{H}$ & -2.042969 & -1.732649 & 0.874636 & 0.000011 & 0.000014 & -0.000124 \\
\hline $15 \mathrm{H}$ & -2.042969 & -1.732649 & -0.874636 & 0.000011 & 0.000014 & 0.000124 \\
\hline $16 \mathrm{H}$ & -0.063036 & 2.511865 & 0.000000 & -0.000112 & 0.000020 & 0.000000 \\
\hline $17 \mathrm{H}$ & -2.500844 & 2.364940 & 0.000000 & -0.000061 & 0.000000 & 0.000000 \\
\hline $18 \mathrm{H}$ & 4.537587 & -1.323772 & 0.000000 & 0.000171 & 0.000014 & 0.000000 \\
\hline $19 \mathrm{H}$ & 4.410457 & 0.212538 & -0.895280 & -0.000007 & -0.000014 & -0.000016 \\
\hline $20 \mathrm{H}$ & 4.410457 & 0.212538 & 0.895280 & -0.000007 & -0.000014 & 0.000016 \\
\hline $21 \mathrm{H}$ & -4.586509 & 1.031197 & 0.000000 & -0.000009 & 0.000012 & 0.000000 \\
\hline $22 \mathrm{H}$ & -4.518439 & -0.829242 & 0.000000 & 0.000211 & 0.000006 & 0.000000 \\
\hline
\end{tabular}

Molecular Energies

Electronic Energies:

Total SCF Energy

$=-479.3426551$ Hartree $-300791.750 \mathrm{kcal} / \mathrm{mol}$

Nuclear Repulsion Energy = 568.4944564 Hartree 


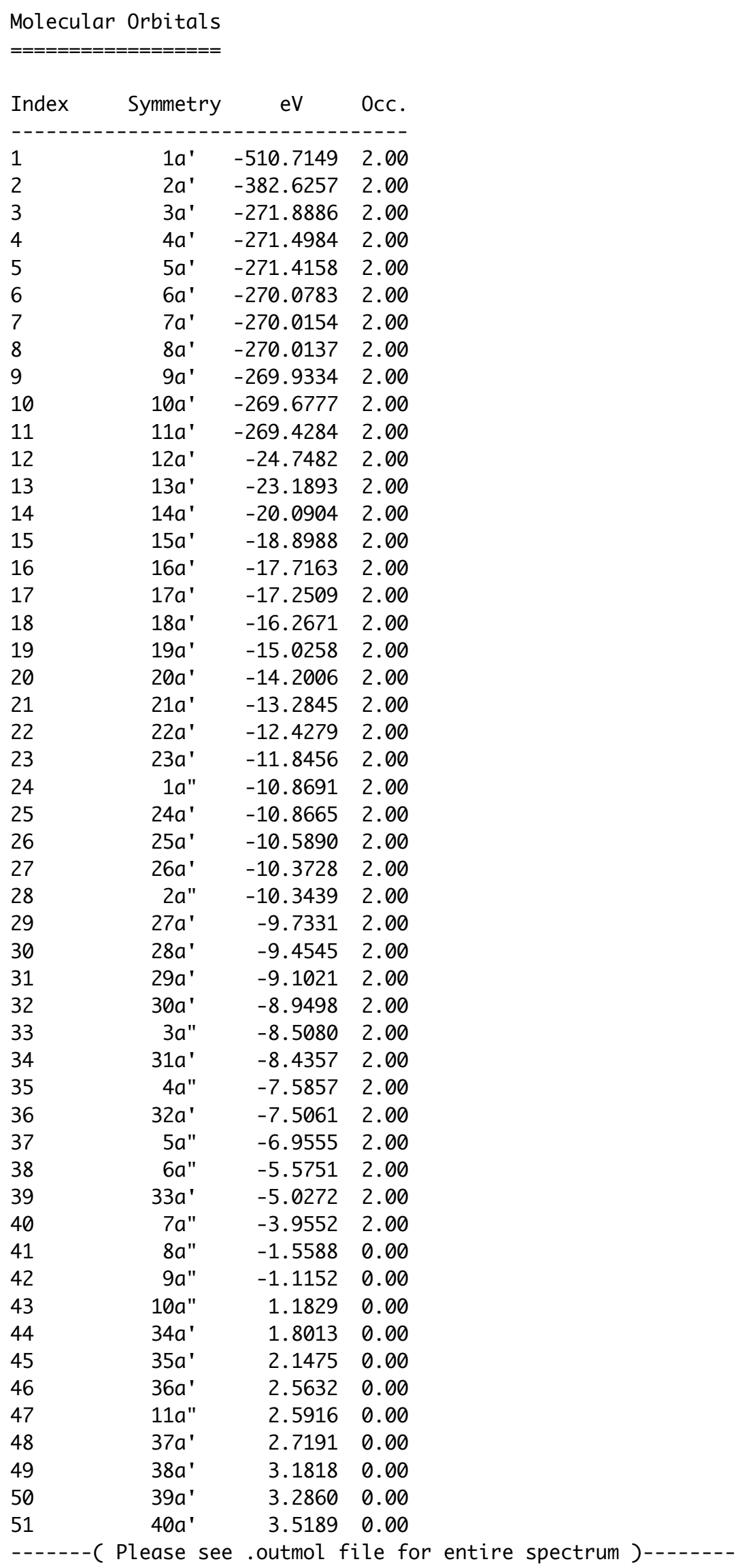


Atom Centered Properties

\begin{tabular}{|c|c|c|c|c|}
\hline \multirow{2}{*}{ Atom } & \multicolumn{4}{|c|}{ Partial Charges (au) } \\
\hline & MUL & LOW & ESP & $\mathrm{RD}$ \\
\hline $1 \mathrm{C}$ & -0.3070 & -0.2618 & -0.0841 & -0.1373 \\
\hline $2 C$ & 0.3473 & 0.1247 & 0.4995 & 0.0329 \\
\hline $3 c$ & 0.0940 & -0.1000 & -0.2493 & -0.0987 \\
\hline $4 C$ & -0.1221 & -0.1506 & -0.0624 & -0.0611 \\
\hline $5 \mathrm{C}$ & -0.1081 & -0.1794 & -0.2882 & -0.0866 \\
\hline $6 \mathrm{C}$ & 0.2075 & 0.0132 & 0.2189 & 0.0572 \\
\hline 70 & -0.5248 & -0.2921 & -0.5301 & -0.2103 \\
\hline $8 \mathrm{H}$ & 0.2849 & 0.2483 & 0.3011 & 0.1253 \\
\hline $9 \mathrm{C}$ & 0.0878 & -0.0589 & 0.0114 & 0.0456 \\
\hline $10 \mathrm{H}$ & 0.0803 & 0.1490 & 0.1386 & 0.0447 \\
\hline $11 \mathrm{~N}$ & -0.4352 & -0.1682 & -0.2050 & 0.0794 \\
\hline $12 \mathrm{C}$ & -0.1559 & -0.3155 & -0.1912 & -0.1216 \\
\hline & -0.2925 & -0.3504 & -0.5015 & -0.1843 \\
\hline $14 \mathrm{H}$ & 0.1289 & 0.1628 & 0.0556 & 0.0834 \\
\hline $15 \mathrm{H}$ & 0.1289 & 0.1628 & 0.0556 & 0.0834 \\
\hline $16 \mathrm{H}$ & 0.0462 & 0.1408 & 0.0882 & 0.0422 \\
\hline $17 \mathrm{H}$ & 0.0443 & 0.1430 & 0.1335 & 0.0431 \\
\hline $18 \mathrm{H}$ & 0.1187 & 0.1577 & 0.1093 & 0.0651 \\
\hline $19 \mathrm{H}$ & 0.1178 & 0.1434 & 0.0908 & 0.0524 \\
\hline $20 \mathrm{H}$ & 0.1178 & 0.1434 & 0.0908 & 0.0524 \\
\hline $21 \mathrm{H}$ & 0.0724 & 0.1443 & 0.1615 & 0.0464 \\
\hline $22 \mathrm{H}$ & 0.0688 & 0.1436 & 0.1570 & 0.0462 \\
\hline
\end{tabular}

Molecular Properties

Electrostatic Moments

Dipole Moment (Debye)
$<\mathrm{x}>\mathrm{B} \quad 3.149476 \quad<\mathrm{y}>\mathrm{:} \quad 1.326843 \quad<\mathrm{z}>\mathrm{:} 0.000000$
3.4176 Debye

Non-Zero Cartesian Moments (Debye/Angstrom units)

$\begin{array}{rrrrrr}r x x>: & -52.188568 & <y y>: & -66.170675 & <z z>: & -67.302203 \\ <x y>: & 1.738940 & <x x x>: & 83.461289 & <y y y>: & 27.666663 \\ <x x y>: & -5.078003 & <y y x>: & 7.467866 & <z z x>: & 13.419463 \\ <z z y>: & 0.085216 & <x x x x>: & -2148.657927 & <y y y y>: & -554.617547 \\ <z z z z>: & -79.596944 & <x x x y>: & 55.208012 & <y y y x>: & 80.878402 \\ <x x y y>: & -438.906188 & <x x z z>: & -424.124530 & <y y z z>: & -105.095764 \\ <z z x y>: & 35.761351 & & & \end{array}$


Product: turbomole

Job Name: z_isomer_finalo

Calculation Type: Optimize

Created: Thu Dec 16 16:06:01 2004
Method: dft
Basis: 6-31g**
Symmetry: cs
Spin: RESTRICTED
Charge: 0
Multiplicity: 1
Functionals: blyp Grid: xfine

\#\#\#\#\#\#\#\#\#\#\#\#\#\#\#\#\#\#\#\#\#\#\#\#\#\#\#\#\#\#\#\#\#\#\#\#\#\#\#\#\#\#\#\#\#\#\#\#\#\#\#\#\#\#\#\#\#\#\#\#\# \#\#\#\#

Optimized Molecular Geometry

Cartesian Coordinates (Angstrom)

\begin{tabular}{|c|c|c|c|}
\hline Atom & $x$ & $y$ & z \\
\hline \multicolumn{4}{|l|}{----} \\
\hline $1 C$ & -1.796524 & -1.095265 & 0.000000 \\
\hline $2 C$ & -0.261454 & -0.973990 & 0.000000 \\
\hline $3 c$ & 0.339009 & 0.358959 & 0.000000 \\
\hline $4 C$ & -0.529532 & 1.529659 & 0.000000 \\
\hline $5 \mathrm{C}$ & -1.893378 & 1.461636 & 0.000000 \\
\hline $6 \mathrm{C}$ & -2.622060 & 0.198450 & 0.000000 \\
\hline 70 & 0.415751 & -2.03 & 0.000000 \\
\hline $8 \mathrm{C}$ & 1.7 & 0.50 & 0000 \\
\hline $9 \mathrm{~N}$ & 2.608791 & -0.523906 & 0.000000 \\
\hline $10 \mathrm{C}$ & 4.061445 & -0.365171 & 0.000000 \\
\hline $1 \mathrm{C}$ & -3.980 & 0.15 & 0.000000 \\
\hline $2 \mathrm{H}$ & -2.056293 & -1.717250 & 0.875074 \\
\hline $13 \mathrm{H}$ & -2.056293 & -1.717250 & -0.875074 \\
\hline $14 \mathrm{H}$ & -0.042933 & 2.511683 & 0.000000 \\
\hline $15 \mathrm{H}$ & -2.481757 & 2.383548 & 0.000000 \\
\hline $16 \mathrm{H}$ & 2.168896 & 1.508279 & 0.000000 \\
\hline $17 \mathrm{H}$ & 2.149296 & -1.451174 & 0.000000 \\
\hline $8 \mathrm{H}$ & 4.524873 & -1.360551 & 0.000000 \\
\hline $19 \mathrm{H}$ & 4.413226 & 0.177259 & -0.895155 \\
\hline $20 \mathrm{H}$ & 4.413226 & 0.177259 & 0.895155 \\
\hline & -4.576645 & 1.069353 & 0.000000 \\
\hline & -4.528858 & -0.791341 & 0.000000 \\
\hline
\end{tabular}

Cartesian Gradients (au)

$\mathrm{dE} / \mathrm{dx} \quad \mathrm{dE} / \mathrm{dy} \quad \mathrm{dE} / \mathrm{dz}$

$-0.000017-0.000010$

0.000000

$0.000007 \quad 0.000002$

0.000000

$-0.000035-0.000001$

0.000000

$0.000041-0.000025$

0.000000

$-0.000024-0.000007$

0.000000

$0.000014-0.000010$

0.000000

0.0000090 .000013

0.000000

$0.000046 \quad 0.000012$

0.000000

$-0.000045-0.000011$

0.000000

$0.000076 \quad 0.000011$

0.000000

$-0.000023 \quad 0.000007$

0.000000

$-0.000001$

0.000001

0.000006

$-0.000001$

0.000001

$-0.000006$

$-0.000005$

0.000008

0.000000

0.000002

0.000011

0.000000

$-0.000006-0.000020$

0.000000

$-0.000002$

0.000009

0.000000

$\begin{array}{llll}-0.000037 & 0.000027 & 0.000000\end{array}$

$-0.000004-0.000009 \quad 0.000042$

$-0.000004-0.000009-0.000042$

$\begin{array}{lll}0.000010 & 0.000012 & 0.000000\end{array}$

$0.000002-0.000014 \quad 0.000000$ 
Molecular Energies

Electronic Energies:

Total SCF Energy

$=-479.3426567$ Hartree

$-300791.751 \mathrm{kcal} / \mathrm{mol}$

Nuclear Repulsion Energy

$=568.4760157$ Hartree

Molecular Orbitals

\begin{tabular}{|c|c|c|c|}
\hline Index & Symmetry & $\mathrm{eV}$ & 0cc. \\
\hline 1 & $1 a^{\prime}$ & -510.7162 & 2.00 \\
\hline 2 & $2 a^{\prime}$ & -382.6249 & 2.00 \\
\hline 3 & $3 a^{\prime}$ & -271.8899 & 2.00 \\
\hline 4 & $4 a^{\prime}$ & -271.4986 & 2.00 \\
\hline 5 & $5 a^{\prime}$ & -271.4151 & 2.00 \\
\hline 6 & $6 a^{\prime}$ & -270.0804 & 2.00 \\
\hline 7 & $7 a^{\prime}$ & -270.0163 & 2.00 \\
\hline 8 & $8 a^{\prime}$ & -270.0139 & 2.00 \\
\hline 9 & $9 a^{\prime}$ & -269.9324 & 2.00 \\
\hline 10 & $10 a^{\prime}$ & -269.6768 & 2.00 \\
\hline 11 & $11 a^{\prime}$ & -269.4301 & 2.00 \\
\hline 12 & $12 a^{\prime}$ & -24.7495 & 2.00 \\
\hline 13 & $13 a^{\prime}$ & -23.1889 & 2.00 \\
\hline 14 & $14 a^{\prime}$ & -20.0902 & 2.00 \\
\hline 15 & $15 a^{\prime}$ & -18.8991 & 2.00 \\
\hline 16 & $16 a^{\prime}$ & -17.7166 & 2.00 \\
\hline 17 & $17 a^{\prime}$ & -17.2494 & 2.00 \\
\hline 18 & $18 a^{\prime}$ & -16.2662 & 2.00 \\
\hline 19 & $19 a^{\prime}$ & -15.0253 & 2.00 \\
\hline 20 & $20 a^{\prime}$ & -14.1993 & 2.00 \\
\hline 21 & $21 a^{\prime}$ & -13.2854 & 2.00 \\
\hline 22 & $22 a^{\prime}$ & -12.4273 & 2.00 \\
\hline 23 & $23 a^{\prime}$ & -11.8454 & 2.00 \\
\hline 24 & $1 a^{\prime \prime}$ & -10.8689 & 2.00 \\
\hline 25 & $24 a^{\prime}$ & -10.8659 & 2.00 \\
\hline 26 & $25 a^{\prime}$ & -10.5894 & 2.00 \\
\hline 27 & $26 a^{\prime}$ & -10.3730 & 2.00 \\
\hline 28 & $2 a^{\prime \prime}$ & -10.3441 & 2.00 \\
\hline 29 & $27 a^{\prime}$ & -9.7327 & 2.00 \\
\hline 30 & $28 a^{\prime}$ & -9.4550 & 2.00 \\
\hline 31 & $29 a^{\prime}$ & -9.1028 & 2.00 \\
\hline
\end{tabular}




$\begin{array}{lrrr}32 & 30 a^{\prime} & -8.9480 & 2.00 \\ 33 & 3 a^{\prime \prime} & -8.5094 & 2.00 \\ 34 & 31 a^{\prime} & -8.4354 & 2.00 \\ 35 & 4 a^{\prime \prime} & -7.5862 & 2.00 \\ 36 & 32 a^{\prime} & -7.5055 & 2.00 \\ 37 & 5 a^{\prime \prime} & -6.9555 & 2.00 \\ 38 & 6 a^{\prime \prime} & -5.5756 & 2.00 \\ 39 & 33 a^{\prime} & -5.0280 & 2.00 \\ 40 & 7 a^{\prime \prime} & -3.9544 & 2.00 \\ 41 & 8 a^{\prime \prime} & -1.5591 & 0.00 \\ 42 & 9 a^{\prime \prime} & -1.1150 & 0.00 \\ 43 & 10 a^{\prime \prime} & 1.1815 & 0.00 \\ 44 & 34 a^{\prime} & 1.8005 & 0.00 \\ 45 & 35 a^{\prime} & 2.1467 & 0.00 \\ 46 & 36 a^{\prime} & 2.5628 & 0.00 \\ 47 & 11 a^{\prime \prime} & 2.5928 & 0.00 \\ 48 & 37 a^{\prime} & 2.7191 & 0.00 \\ 49 & 38 a^{\prime} & 3.1823 & 0.00 \\ 50 & 39 a^{\prime} & 3.2871 & 0.00 \\ 51 & 40 a^{\prime} & 3.5211 & 0.00 \\ ----(\text { Please see } & .04 t m o l \text { file for entire spectrum )------- }\end{array}$

Atom Centered Properties

\begin{tabular}{|c|c|c|c|c|}
\hline Atom & \multicolumn{4}{|c|}{ Partial Charges (au) } \\
\hline & MUL & LOW & ESP & RD \\
\hline $1 \mathrm{C}$ & -0.3071 & -0.2617 & -0.0549 & -0.1373 \\
\hline $2 c$ & 0.3473 & 0.1248 & 0.4953 & 0.0324 \\
\hline $3 c$ & 0.0941 & -0.1000 & -0.2532 & -0.0985 \\
\hline $4 \mathrm{C}$ & -0.1220 & -0.1506 & -0.0581 & -0.0610 \\
\hline $5 \mathrm{C}$ & -0.1083 & -0.1796 & -0.2867 & -0.0866 \\
\hline $6 \mathrm{C}$ & 0.2076 & 0.0132 & 0.2076 & 0.0572 \\
\hline 70 & -0.5247 & -0.2920 & -0.5311 & -0.2098 \\
\hline $8 \mathrm{C}$ & 0.0877 & -0.0588 & 0.0111 & 0.0456 \\
\hline $9 \mathrm{~N}$ & -0.4353 & -0.1681 & -0.2043 & 0.0795 \\
\hline $10 \mathrm{C}$ & -0.1558 & -0.3155 & -0.1832 & -0.1216 \\
\hline $11 \mathrm{C}$ & -0.2925 & -0.3503 & -0.4990 & -0.1843 \\
\hline $12 \mathrm{H}$ & 0.1289 & 0.1628 & 0.0479 & 0.0834 \\
\hline $13 \mathrm{H}$ & 0.1289 & 0.1628 & 0.0479 & 0.0834 \\
\hline $14 \mathrm{H}$ & 0.0462 & 0.1407 & 0.0862 & 0.0422 \\
\hline $15 \mathrm{H}$ & 0.0442 & 0.1430 & 0.1330 & 0.0431 \\
\hline $16 \mathrm{H}$ & 0.0802 & 0.1489 & 0.1390 & 0.0447 \\
\hline $17 \mathrm{H}$ & 0.2850 & 0.2481 & 0.3009 & 0.1253 \\
\hline $18 \mathrm{H}$ & 0.1187 & 0.1578 & 0.1070 & 0.0651 \\
\hline $19 \mathrm{H}$ & 0.1178 & 0.1433 & 0.0885 & 0.0524 \\
\hline $20 \mathrm{H}$ & 0.1178 & 0.1433 & 0.0885 & 0.0524 \\
\hline
\end{tabular}




$\begin{array}{ccccc}21 \mathrm{H} & 0.0725 & 0.1443 & 0.1614 & 0.0465 \\ 22 \mathrm{H} & 0.0688 & 0.1435 & 0.1564 & 0.0462 \\ -\end{array}$

Molecular Properties

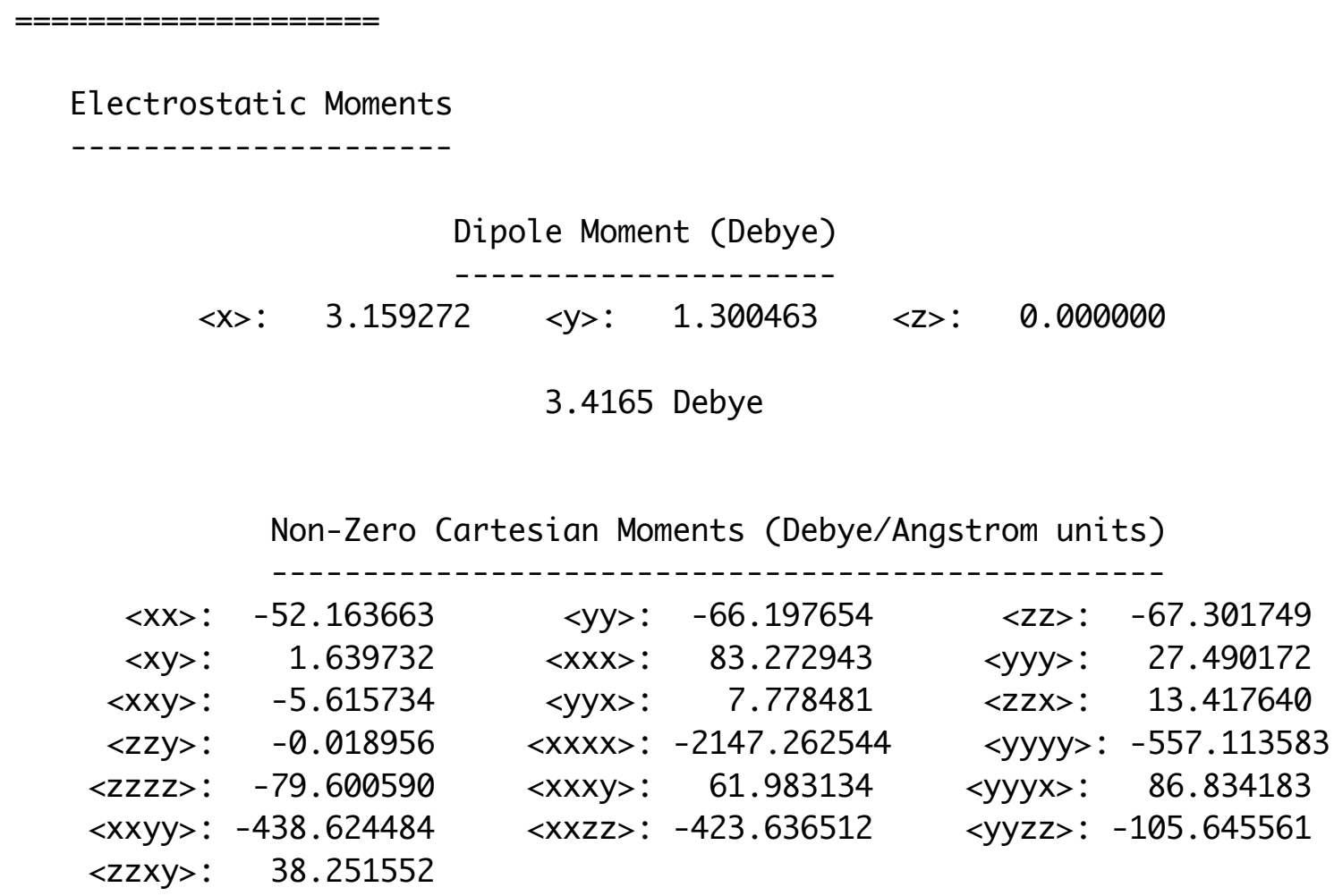

Job Statistics

Thu Dec 16 16:06:01 AEDT 2004

Computer: el-toro.vislab.mq.edu.au CPU: irix6m4

Optimization Cycles: 3

Time: 19932.1u 182.1s 6:22:24 
Product: turbomole

Job Name: e_isomer_finalo

Calculation Type: Optimize

Created: Sat Dec 18 01:23:10 2004
Method: dft
Basis: 6-31g**
Symmetry: cs
Spin: RESTRICTED
Charge: 0
Multiplicity: 1
Functionals: blyp Grid: xfine

\#\#\#\#\#\#\#\#\#\#\#\#\#\#\#\#\#\#\#\#\#\#\#\#\#\#\#\#\#\#\#\#\#\#\#\#\#\#\#\#\#\#\#\#\#\#\#\#\#\#\#\#\#\#\#\#\#\#\#\#\# \#\#\#\#

Optimized Molecular Geometry

Cartesian Coordinates (Angstrom)

\begin{tabular}{|c|c|c|c|}
\hline Atom & $x$ & $y$ & z \\
\hline & & & \\
\hline C & -1.708780 & -1.676412 & 0.000000 \\
\hline $2 C$ & -0.173565 & -1.498985 & 0.000000 \\
\hline C & 0.367456 & -0.118146 & 0.000000 \\
\hline C & -0.556176 & 1.006271 & 0.000000 \\
\hline C & -1.917047 & 0.872243 & 0.000000 \\
\hline $6 \mathrm{C}$ & -2.587985 & -0.420900 & 0.000000 \\
\hline 70 & 0.543 & -2.5 & 0.1 \\
\hline $8 \mathrm{C}$ & 1.75 & -0.0 & 000 \\
\hline $\mathrm{N}$ & 2.494342 & 1.131 & 00000 \\
\hline C & 3.959631 & 1.13 & 0.000000 \\
\hline C & -3.943513 & -0.51 & 0.000000 \\
\hline $\mathrm{H}$ & -1.943905 & -2.307658 & 0.875067 \\
\hline $\mathrm{H}$ & -1.943905 & -2.307658 & 75067 \\
\hline $\mathrm{H}$ & -0.143216 & 2.022332 & 0.000000 \\
\hline $\mathrm{H}$ & -2.547338 & 1.766500 & 0.000000 \\
\hline $\mathrm{H}$ & 2.317874 & -0.943373 & 0.000000 \\
\hline $\mathrm{H}$ & 2.018600 & 2.029296 & 0.000000 \\
\hline $\mathrm{H}$ & 4.311674 & 2.179112 & 0.000000 \\
\hline $\mathrm{H}$ & 4.366383 & 0.637774 & 0.895282 \\
\hline & 4.366383 & 0.637774 & -0.895282 \\
\hline $\mathrm{H}$ & -4.576238 & 0.373834 & 0.000000 \\
\hline & -4.454807 & -1.483355 & 0.000000 \\
\hline
\end{tabular}

Cartesian Gradients (au)

$\mathrm{dE} / \mathrm{dx} \quad \mathrm{dE} / \mathrm{dy} \quad \mathrm{dE} / \mathrm{dz}$

$-0.000024 \quad 0.000022$

0.000000

$-0.000004 \quad 0.000043$

0.000000

$0.000028-0.000027$

0.000000

$-0.000096 \quad 0.000067$

0.000000

$0.000044-0.000102$

0.000000

$-0.000036 \quad 0.000019$

0.000000

$-0.000009 \quad 0.000007$

0.000000

$0.000023-0.000042$

0.000000

$\begin{array}{ll}-0.000060 & 0.000064\end{array}$

0.000000

$-0.000044-0.000043$

0.000000

$0.000251-0.000011$

0.000000

$-0.000001-0.000005-0.000005$

$-0.000001-0.000005 \quad 0.000005$

$-0.000018-0.000047$

0.000000

$0.000003 \quad 0.000041$

0.000000

$-0.000002 \quad 0.000018$

0.000000

$0.000019-0.000041$

0.000000

$\begin{array}{llll}0.000044 & 0.000013 & 0.000000\end{array}$

$\begin{array}{llll}0.000019 & 0.000008 & 0.000007\end{array}$

$\begin{array}{llll}0.000019 & 0.000008 & -0.000007\end{array}$

$\begin{array}{lll}-0.000092 & 0.000042 & 0.000000\end{array}$

$\begin{array}{lll}-0.000054 & -0.000028 & 0.000000\end{array}$ 
Molecular Energies

Electronic Energies:

Total SCF Energy

$=-479.3319898$ Hartree

$-300785.058 \mathrm{kcal} / \mathrm{mol}$

Nuclear Repulsion Energy

$=561.6803645$ Hartree

Molecular Orbitals

\begin{tabular}{|c|c|c|c|}
\hline Index & Symmetry & $\mathrm{eV}$ & 0cc. \\
\hline 1 & $1 a^{\prime}$ & -510.2926 & 2.00 \\
\hline 2 & $2 a^{\prime}$ & -382.9783 & 2.00 \\
\hline 3 & $3 a^{\prime}$ & -271.6874 & 2.00 \\
\hline 4 & $4 a^{\prime}$ & -271.5487 & 2.00 \\
\hline 5 & $5 a^{\prime}$ & -271.2941 & 2.00 \\
\hline 6 & $6 a^{\prime}$ & -270.1297 & 2.00 \\
\hline 7 & $7 a^{\prime}$ & -270.1227 & 2.00 \\
\hline 8 & $8 a^{\prime}$ & -269.9161 & 2.00 \\
\hline 9 & $9 a^{\prime}$ & -269.8429 & 2.00 \\
\hline 10 & $10 a^{\prime}$ & -269.8339 & 2.00 \\
\hline 11 & $11 a^{\prime}$ & -269.5283 & 2.00 \\
\hline 12 & $12 a^{\prime}$ & -24.4488 & 2.00 \\
\hline 13 & $13 a^{\prime}$ & -23.3997 & 2.00 \\
\hline 14 & $14 a^{\prime}$ & -20.0746 & 2.00 \\
\hline 15 & $15 a^{\prime}$ & -18.9029 & 2.00 \\
\hline 16 & $16 a^{\prime}$ & -17.7202 & 2.00 \\
\hline 17 & $17 a^{\prime}$ & -17.2063 & 2.00 \\
\hline 18 & $18 a^{\prime}$ & -16.4967 & 2.00 \\
\hline 19 & $19 a^{\prime}$ & -15.1689 & 2.00 \\
\hline 20 & $20 a^{\prime}$ & -14.1663 & 2.00 \\
\hline 21 & $21 a^{\prime}$ & -13.1914 & 2.00 \\
\hline 22 & $22 a^{\prime}$ & -12.5051 & 2.00 \\
\hline 23 & $23 a^{\prime}$ & -11.9255 & 2.00 \\
\hline 24 & $24 a^{\prime}$ & -10.9639 & 2.00 \\
\hline 25 & $1 a^{\prime \prime}$ & -10.9508 & 2.00 \\
\hline 26 & $25 a^{\prime}$ & -10.6459 & 2.00 \\
\hline 27 & $2 a^{\prime \prime}$ & -10.2409 & 2.00 \\
\hline 28 & $26 a^{\prime}$ & -10.2350 & 2.00 \\
\hline 29 & $27 a^{\prime}$ & -9.8465 & 2.00 \\
\hline 30 & $28 a^{\prime}$ & -9.3307 & 2.00 \\
\hline 31 & $29 a^{\prime}$ & -9.0977 & 2.00 \\
\hline
\end{tabular}




$\begin{array}{lrrr}32 & 30 a^{\prime} & -8.8368 & 2.00 \\ 33 & 3 a^{\prime \prime} & -8.3923 & 2.00 \\ 34 & 31 a^{\prime} & -8.3629 & 2.00 \\ 35 & 4 a^{\prime \prime} & -7.5653 & 2.00 \\ 36 & 32 a^{\prime} & -7.4799 & 2.00 \\ 37 & 5 a^{\prime \prime} & -7.0172 & 2.00 \\ 38 & 6 a^{\prime \prime} & -5.5097 & 2.00 \\ 39 & 33 a^{\prime} & -4.6500 & 2.00 \\ 40 & 7 a^{\prime \prime} & -4.0275 & 2.00 \\ 41 & 8 a^{\prime \prime} & -1.4686 & 0.00 \\ 42 & 9 a^{\prime \prime} & -1.0619 & 0.00 \\ 43 & 34 a^{\prime} & 0.8282 & 0.00 \\ 44 & 10 a^{\prime \prime} & 1.1582 & 0.00 \\ 45 & 35 a^{\prime} & 1.9302 & 0.00 \\ 46 & 36 a^{\prime} & 2.6177 & 0.00 \\ 47 & 37 a^{\prime} & 2.6548 & 0.00 \\ 48 & 11 a^{\prime \prime} & 2.6671 & 0.00 \\ 49 & 38 a^{\prime} & 3.0049 & 0.00 \\ 50 & 39 a^{\prime} & 3.2859 & 0.00 \\ 51 & 40 a^{\prime} & 3.4589 & 0.00 \\ ----(\text { Please see } & .04 t m o l \text { file for entire spectrum )------- }\end{array}$

Atom Centered Properties

\begin{tabular}{|c|c|c|c|c|}
\hline Atom & \multicolumn{4}{|c|}{ Partial Charges (au) } \\
\hline & MUL & LOW & ESP & $\mathrm{RD}$ \\
\hline $1 \mathrm{C}$ & -0.3149 & -0.2669 & -0.1293 & -0.1472 \\
\hline $2 c$ & 0.3386 & 0.1186 & 0.5092 & 0.0483 \\
\hline $3 c$ & 0.0852 & -0.1028 & -0.2476 & -0.1042 \\
\hline $4 \mathrm{C}$ & -0.1199 & -0.1612 & -0.0598 & -0.0746 \\
\hline $5 \mathrm{C}$ & -0.1001 & -0.1680 & -0.2789 & -0.0730 \\
\hline $6 \mathrm{C}$ & 0.2084 & 0.0136 & 0.2352 & 0.0586 \\
\hline 70 & -0.4914 & -0.2699 & -0.5104 & -0.1965 \\
\hline $8 \mathrm{C}$ & 0.0837 & -0.0558 & -0.0013 & 0.0472 \\
\hline $9 \mathrm{~N}$ & -0.4488 & -0.1880 & -0.2192 & 0.0516 \\
\hline $10 \mathrm{C}$ & -0.1515 & -0.3138 & -0.1989 & -0.1155 \\
\hline $11 \mathrm{C}$ & -0.2886 & -0.3438 & -0.4945 & -0.1756 \\
\hline $12 \mathrm{H}$ & 0.1286 & 0.1628 & 0.0644 & 0.0824 \\
\hline $13 \mathrm{H}$ & 0.1286 & 0.1628 & 0.0644 & 0.0824 \\
\hline $14 \mathrm{H}$ & 0.0272 & 0.1325 & 0.0831 & 0.0365 \\
\hline $15 \mathrm{H}$ & 0.0450 & 0.1420 & 0.1301 & 0.0430 \\
\hline $16 \mathrm{H}$ & 0.1104 & 0.1640 & 0.1524 & 0.0569 \\
\hline $17 \mathrm{H}$ & 0.2549 & 0.2370 & 0.2797 & 0.1148 \\
\hline $18 \mathrm{H}$ & 0.1086 & 0.1545 & 0.1031 & 0.0634 \\
\hline $19 \mathrm{H}$ & 0.1253 & 0.1464 & 0.0999 & 0.0538 \\
\hline $20 \mathrm{H}$ & 0.1253 & 0.1464 & 0.0999 & 0.0538 \\
\hline
\end{tabular}




$\begin{array}{ccccc}21 \mathrm{H} & 0.0733 & 0.1440 & 0.1597 & 0.0464 \\ 22 \mathrm{H} & 0.0723 & 0.1455 & 0.1588 & 0.0475 \\ \end{array}$

Molecular Properties

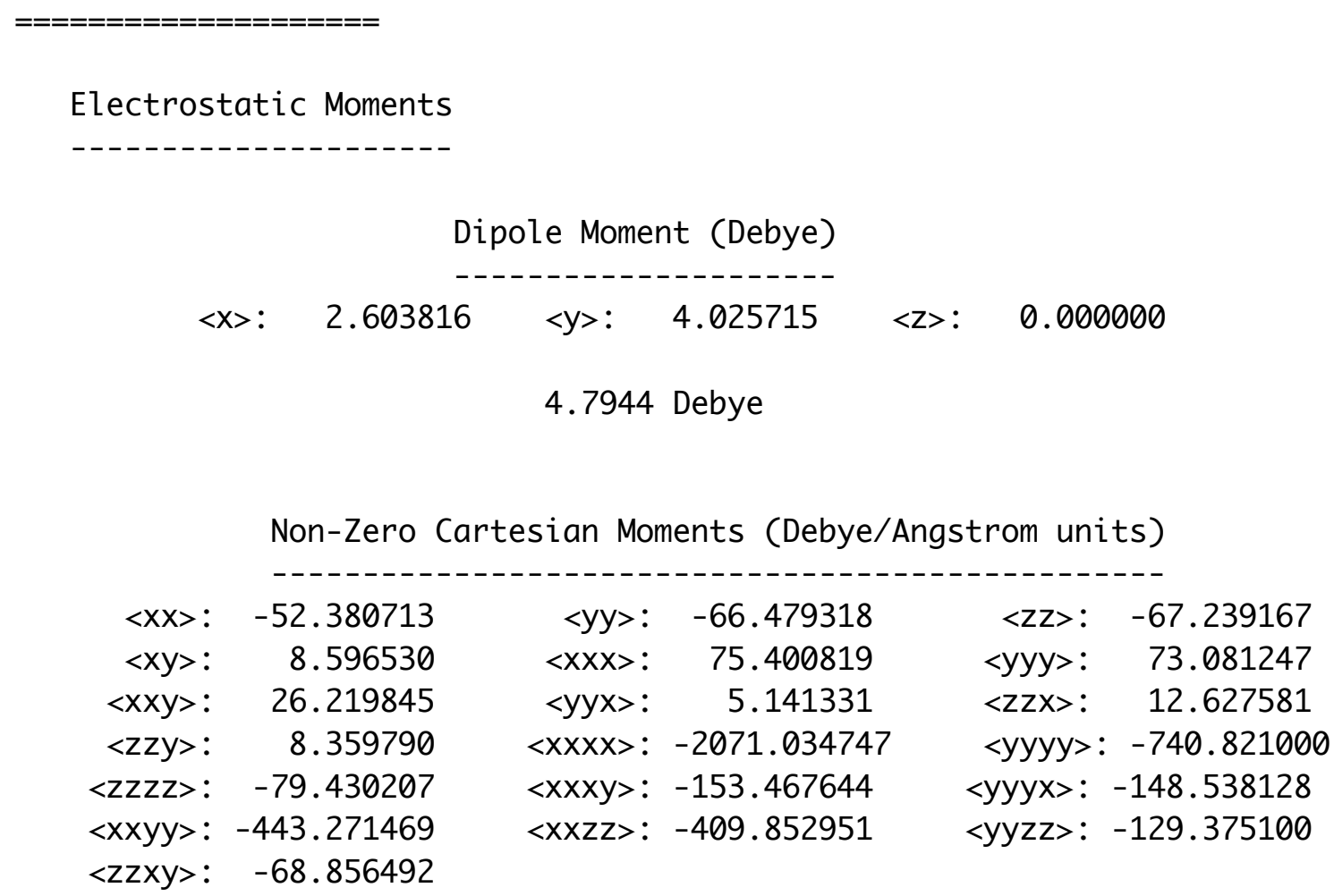

Job Statistics

Sat Dec 18 01:23:10 AEDT 2004

Computer: el-toro.vislab.mq.edu.au CPU: irix6m4

Optimization Cycles: 4

Time: 22979.4u 183.6s 7:19:48 